\title{
$A b$ initio treatment of noncollinear magnets with the full-potential linearized augmented plane wave method
}

\author{
$\mathrm{Ph}$. Kurz and F. Förster \\ Institut für Festkörperforschung, Forschungszentrum Jülich, D-52425 Jülich, Germany \\ L. Nordström \\ Department of Physics, Uppsala University, Box 530, S-751 21 Uppsala, Sweden \\ G. Bihlmayer* and S. Blügel \\ Institut für Festkörperforschung, Forschungszentrum Jülich, D-52425 Jülich, Germany \\ (Received 15 August 2003; published 27 January 2004)
}

\begin{abstract}
The massively parallelized full-potential linearized augmented plane-wave bulk and film program FLEUR for first-principles calculations in the context of density functional theory was adapted to allow calculations of materials with complex magnetic structures-i.e., with noncollinear spin arrangements and incommensurate spin spirals. The method developed makes no shape approximation to the charge density and works with the continuous vector magnetization density in the interstitial and vacuum region and a collinear magnetization density in the spheres. We give an account of the implementation. Important technical aspects, such as the formulation of a constrained local moment method in a full-potential method that works with a vector magnetization density to deal with specific preselected nonstationary-state spin configurations, the inclusion of the generalized gradient approximation in a noncollinear framework, and the spin-relaxation method are discussed. The significance and validity of different approximations are investigated. We present examples to the various strategies to explore the magnetic ground state, metastable states, and magnetic phase diagrams by relaxation of spin arrangements or by performing calculations for constraint spin configurations to invest the functional dependence of the total energy and magnetic moment with respect to external parameters.
\end{abstract}

DOI: 10.1103/PhysRevB.69.024415 PACS number(s): 75.70.Ak, 71.15.Ap, 75.30.Fv, 71.15.Mb

\section{INTRODUCTION}

Noncollinear magnetism in general and incommensurate spin-density waves in particular are complex magnetic structures which exist in a variety of systems. They often occur for topologically frustrated antiferromagnets, such as antiferromagnets on a triangular lattice, disordered systems, exchange bias systems, and molecular magnets, or for systems which exhibit either competing exchange interactions such as, for example, for fcc Fe, the lanthanides, and multicomponent magnets-e.g., $\mathrm{LaMn}_{2} \mathrm{Ge}_{2}$ - or exhibit competition between exchange and spin-orbit interactions as for $\mathrm{U}_{3} \mathrm{P}_{4}$. Noncollinear magnetism occurs in spin-glass systems and in domain walls and is natural for spin fluctuations at finite temperature. ${ }^{1}$

In 1972, von Barth and Hedin ${ }^{2}$ introduced the spinpolarized density functional theory (DFT). Already at that time, the key quantity of the theory, the magnetization density, was introduced as a continuous three-dimensional vector field without any limitation to its local direction. Thus it included already the treatment of complex magnetic structures. Although noncollinear magnetism is a widespread phenomenon, even today by far the majority of all $a b$ initio methods available are restricted to collinear magnetic systems. This restriction has the advantage that the Hamiltonian is diagonal in spin space. This does not only save a huge amount of computer time, but it also greatly simplifies the implementation of magnetism into an existing non-spinpolarized program, because the spin-up and spin-down prob- lem can be treated almost independently, almost like two nonmagnetic calculations.

The first self-consistent noncollinear ab initio calculations for periodic solids-e.g., Refs. 3-5-applied the so-called atomic sphere approximation (ASA) to the magnetization density. In this approximation everywhere inside each Wigner-Seitz sphere the magnetization density is spherically symmetric and the direction is kept the same (collinear). Only interatomic noncollinearity between the magnetic moments at different atomic sites was allowed in these calculations. This is consistent with the intuitive picture that each atom carries a magnetic moment and these moments and their directions differ only between the atoms. Such methods are very suitable to describe the interatomic noncollinearity of close-packed systems. There are, however, problems which call one to go beyond the atomic sphere type of approximation. One of those problems is the neglect of the intra-atomic noncollinearity, which occurs, for example, due to the competition of the exchange interaction and spin-orbit interaction in systems with large relativistic effects. A second class of problems arises if one deals with noncollinear magnetism in systems with low symmetry or in fairly open structures for which the atomic sphere approximation is known to be less suitable and often provides results with insufficient accuracy. Very recently several groups ${ }^{6-12}$ developed fully unconstrained noncollinear $a b$ initio programs that treat the magnetization density as a continuous vector field.

The aim of the present work is to introduce a noncollinear $a b$ initio method developed on the basis of the full-potential linearized augmented plane-wave (FLAPW) concept. It is 
designed to study complex magnetic structures in systems with low symmetries, open geometries, or reduced dimensions. It allows for total energy and force calculations to simultaneously optimize the atomic and magnetic structure. We adopted a "hybrid" approach, treating the magnetization density (as well as the charge density and potential) without any shape approximation and treating the magnetization density as a continuous vector field everywhere, except within nonoverlapping spheres around each atomic site. For transition metals the noncollinearity in these spheres is expected to be small, and we show that this indeed is a very good approximation for these systems. This is already anticipated in the concept of assigning a direction to the magnetic moment of a specific atom. Several strategies are implemented to deal with a large class of complex magnetic states.

An arbitrary magnetic configuration (i.e., a chosen arrangement of directions of local magnetic moments) is in general not a stationary state of the magnetic system. Requiring that the magnetic moments assemble in a prescribed configuration means to constrain the phase space of possible solutions. Within the framework of DFT such a constraint is taken into account by a set of Lagrange parameters. The Lagrange parameter represents a "constraining force" or magnetic constraint field applied to the magnetic moments of the atoms to keep the system in the desired magnetic configuration. We have implemented the constrained local moment method in a "full-potential" program that works with the vector magnetization density. We will show in Sec. II B that this implementation allows us to test the functional form of the energy and magnetization with respect to external parameters-i.e., the angles specifying the directions of the local magnetic moments around the atom-and compare the results with model Hamiltonians. We note that this approach is very powerful, as deviations from the anticipated forms of the energy as a function of these external parameters can lead to important and far-reaching conclusions. ${ }^{13,14}$

The constraint fields can also be interpreted as a torque which can be used to persue adiabatic spin dynamics. ${ }^{15,16} \mathrm{We}$ did not follow this line. Instead we have implemented a spinrelaxation method which allows us to find and test local minima spin structures after a constraint on a given spin structure is released.

An important class of noncollinear configurations is spiral magnetic states or spin spirals. These are configurations where the local moment is rotated by a constant angle from atom to atom along a certain direction through the crystal. Spin-spiral states occur as magnetic ground states in nature-e.g., in fcc Fe (Ref. 17), in rare-earth metals, and frequently in multicomponent magnets with competing magnetic interactions-e.g., $\mathrm{LaMn}_{2} \mathrm{Ge}_{2} \cdot{ }^{18}$ Spin spirals can also be understood as a model for magnons or domain walls. When spin-orbit coupling is neglected, a generalization of the Bloch theorem for spiral magnetic configurations can be derived. On the basis of this generalized Bloch theorem, we have implemented a method that allows us to deal with spinspiral states using only the chemical unit cell of the crystal without the need for large supercells (Sec. II C). Incommensurate spin spirals can never be described with supercells. With the implementation of noncollinear magnetism, spin spirals, the local spin-relaxation method, and the constrained local moment method, into a bulk and film FLAPW program, we have developed a unique tool to investigate noncollinearity in bulk and in particular at magnetic surfaces, in thin films and low-dimensional magnets in general.

Compared with nonmagnetic or collinear magnetic calculations the computational effort of noncollinear calculations is enormous. Since the spin-up and spin-down problems cannot be solved separately anymore, the size of the Hamiltonian matrix that has to be diagonalized doubles. In addition, also for calculations of structures with inversion symmetry the matrix becomes complex Hermitian rather than real symmetric. In most cases the noncollinear magnetic structures have a lower symmetry, and thus large irreducible Brillouine zones, and have often also a larger unit cell containing more atoms than the collinear configuration. Small energy differences between competing magnetic states require calculations with high computational precision and require thus a very good sampling of the Brillouin-zone integrals by choosing a large number of $k$ points. Therefore, noncollinear $a b$ initio calculations represent a cutting edge problem in supercomputing. Without a parallelized version of the program and access to massively parallel supercomputers most calculations of realistic systems are prohibitively slow.

The principles of noncollinear magnetism, the constrained local moment method, and spin-spiral calculations are described in Sec. II. Practical aspects of the calculations are then given in Sec. III, which also includes tests of the constrained local moment and the spin-spiral method. The implementation of noncollinear magnetism in the FLAPW method is finally described in the Appendix.

\section{METHOD}

\section{A. Noncollinear magnetism}

The energy functional of a general magnetic system can be expressed in two ways. It can be written as a functional of the charge density $n$ and the magnetization density vector field $\mathbf{m}$ or as a functional of the Hermitian $2 \times 2$ density matrix $\boldsymbol{\rho}$. The two formulations are completely equivalent. The density matrix is defined by the following equation:

$$
\boldsymbol{\rho}=\frac{1}{2}\left(n \mathbf{I}_{2}+\boldsymbol{\sigma} \cdot \mathbf{m}\right)=\frac{1}{2}\left(\begin{array}{cc}
n+m_{z} & m_{x}-i m_{y} \\
m_{x}+i m_{y} & n-m_{z}
\end{array}\right),
$$

where $\mathbf{I}_{2}$ is the unit matrix in spin space and $\boldsymbol{\sigma}$ is the vector of the Pauli matrices. We can also define the potential matrix $\mathbf{V}$ in the same way:

$$
\mathbf{V}=V \mathbf{I}_{2}+\mu_{B} \boldsymbol{\sigma} \cdot \mathbf{B} .
$$

$V$ contains the external, Hartree, and the exchangecorrelation potential, averaged over two spin directions in a local frame of reference where the $z$ axis is parallel to the local quantization axis. B comprises the external $B$ field and the exchange field expressed as the difference of exchangecorrelation potential, $1 / 2\left[V_{x c}(\uparrow)-V_{x c}(\downarrow)\right]$, in the local frame of reference parallel to the local quantization axis. 
Introducing $i$ as a composite index for the $\mathbf{k}$ vector and state, $i=(\mathbf{k}, \nu)$, we compare Eq. (1) with

$$
n(\mathbf{r})=\sum_{i=1}^{N} \boldsymbol{\psi}_{i}^{\dagger}(\mathbf{r}) \mathbf{I}_{2} \boldsymbol{\psi}_{i}(\mathbf{r}), \quad \mathbf{m}(\mathbf{r})=\sum_{i=1}^{N} \boldsymbol{\psi}_{i}^{\dagger}(\mathbf{r}) \boldsymbol{\sigma} \boldsymbol{\psi}_{i}(\mathbf{r}),
$$

and see that the components of the density matrix, $\rho_{\alpha \beta}$, are given by a very simple relation in terms of the solutions of the Kohn-Sham equation $\psi_{i}=\left(\psi_{i, 1}, \psi_{i, 2}\right)$ :

$$
\rho_{\alpha \beta}=\sum_{i=1}^{N} \psi_{i, \alpha}^{*} \psi_{i, \beta} \text { with } \alpha, \beta \in 1,2 .
$$

Using the potential matrix [Eq. (2)], the Kohn-Sham equation for a given $\mathbf{k}$-point obtains the form

$$
\left\{-\frac{\hbar^{2}}{2 m} \nabla^{2} \mathbf{I}_{2}+\mathbf{V}\right\} \boldsymbol{\psi}_{\nu}=\epsilon_{\nu} \boldsymbol{\psi}_{\nu}
$$

The kinetic energy part of the Hamiltonian is diagonal in the two-dimensional spin space. It is only the off-diagonal part of the Hermitian $2 \times 2$ potential matrix that couples the two components of the Pauli spinor $\psi_{\nu}$. If the $B$ field is collinear, the spin coordinate frame can always be chosen such that the $B$ field points in the spin $z$ direction.

Since the density functional theory was first proposed, different parametrizations of the exchange correlation energy $\epsilon_{x c}$ have been suggested in the local spin-density approximation (LSDA) and also in the generalized gradient approximation (GGA). These parametrizations have been developed and mostly used for collinear calculations. Due to the local character of the LSDA, $\epsilon_{x c}$ depends only on the magnitude of the magnetization $\left[\epsilon_{x c}=\epsilon_{x c}(n,|\mathbf{m}|)\right]$. Hence, there is no reference to any direction and the LSDA can equally be applied to collinear and noncollinear systems. All that needs to be done is to locally calculate $n$ and $|\mathbf{m}|$ and memorize the local direction of $\mathbf{m}$, since the exchange-correlation $B$ field always has the same direction as the magnetization. After this step the standard parametrizations can be applied. In contrast, in the generalized gradient approximation the environment of a point in space does enter the formula for $\epsilon_{x c}$ through the gradients of the densities. In general, the gradients of $n, m_{x}, m_{y}$, and $m_{z}$ have to be considered. The most wide spread parametrizations are, however, developed for collinear calculations and hence consider only the gradients of the scalar quantities $n$ and $m$. Therefore, these parametrizations are in principle not applicable to a noncollinear system. In practice, the GGA can be used in an approximate way, because the contribution of the gradient of density $n$, $\nabla n$, is more important than the gradients of the magnetization $\mathbf{m}$. There are two possible quantities to feed into the parametrization in replacement of the gradient of $m$ in a collinear calculation: (i) the gradient of the magnitude of the magnetization vector field $\nabla|\mathbf{m}|$ and (ii) the $z$ component of the gradient of the magnetization vector field projected onto the local direction of the magnetization. We will discuss the differences between the two possibilities in Sec. III B 4 .

Noncollinear magnetism has already been implemented into a FLAPW code by Nordström and co-workers. ${ }^{6,11}$ These authors work with more physical quantities such as the density $n(\mathbf{r})$ and the magnetization density $\mathbf{m}(\mathbf{r})$, and with a spin-independent LAPW basis set extended inside the spheres around the atom by spin-dependent local orbitals. The implementation presented here is based on an alternative strategy: namely, on the spin-density matrices and on the standard LAPW basis set using spin-dependent radial wave functions $u_{l \sigma}(r)$ and their energy derivatives $\dot{u}_{l \sigma}(r)$ supplemented by spin-dependent local orbitals. The biggest difference, however, arises from the motivation that most theoretical models dealing with noncollinear magnetism are based on the concept of a (semiclassical) spin associated with an atom and a quantum-mechanical description of the interaction between these spins. The success of these models-e.g., the Heisenberg model-and the usefulness of calculational ab initio methods that - like the augmented spherical-wave (ASW) method-neglect all intra-atomic noncollinearity motivated us to develop a "hybrid" method that assumes a collinear $B$ field in the vicinity of the atomic nuclei and a continuous vector $\mathbf{B}$ field in the interatomic region and in the vacuum.

Therefore, inside nonoverlapping spheres centered around the atoms the off-diagonal elements of the potential matrix $\mathbf{V}$ that enters the Kohn-Sham equation (5) are assumed to be zero in the local coordinate frame of this atom. In this local frame, the magnetic moment of atom $\alpha$ then points in the $z$ direction and this direction can then, in the global frame, be specified by a direction $\hat{\mathbf{e}}_{M}^{\alpha}$. In a normal calculation these directions are not input quantities but have to be determined self-consistently. Alternatively, in a "constrained" calculation (see next section), the $\hat{\mathbf{e}}_{M}^{\alpha}$, s can be used as external parameters to the calculation. As we will give evidence below, the noncollinear magnetism of $3 d$ transition metals at surfaces and in open structures is an example of weak intraatomic noncollinearity. Typically $\mathbf{m}(\mathbf{r})$ is well localized inside the atomic sphere, where $\mathbf{m}(\mathbf{r})$ is essentially parallel to the average of the spin density of the sphere except in regions close to the sphere boundary where $\mathbf{m}(\mathbf{r})$ is already small. Since in the FLAPW method muffin-tin spheres are used which are significantly smaller than volume filling atomic spheres and since the choice of the sphere radii is flexible to a certain degree, but definitely smaller than half the nearest-neighbor distance, a "hybrid" method in which the full magnetization density $\mathbf{m}(\mathbf{r})$ is treated without shape approximation and as a continuous vector field in the interstitial region and in the vacuum, while inside each muffin-tin sphere we only allow for one direction of magnetization that enters the determination of the $\mathbf{B}$ field,

$$
\mathbf{m}(\mathbf{r})= \begin{cases}\mathbf{m}(\mathbf{r}), & \text { interstitial and vacuum } \\ m^{\alpha}(\mathbf{r}) \hat{\mathbf{e}}_{M}^{\alpha}, & \text { muffin-tin sphere } \alpha,\end{cases}
$$

should provide excellent results. The notation here applies to the FLAPW in bulk and in the film geometry, ${ }^{27,28}$ where the space is partitioned into a film of finite thickness, consisting of an interstitial region and muffin-tin spheres $\alpha$ and two semi-indefinite vacuum regions on both sides of the film. The 
continuous vector-field description in the interstitial region describes to a large extent the intra-atomic magnetism of, e.g., $3 d$ metals. The exchange field $\mathbf{B}$ is also expressed according to Eq. (6). The intra-atomic noncollinearity of $\mathbf{m}$ in the sphere for this $\mathbf{B}$ field is also known, but not used in the construction of the $\mathbf{B}$ field.

A detailed account of the implementation of this formalism in the FLAPW method is given in Appendix A.1. All results in the present work have been obtained within the scalar relativistic approximation; i.e. the spin-orbit coupling (SOC) is neglected.

\section{B. Constraint}

With the exception of high-symmetry states, like the ferromagnetic, antiferromagnetic, or other collinear magnetic states, and a certain class of spin-spiral states and particular linear superpositions of several particular spin-spiral states, in general an arbitrary magnetic configuration given by a set of local (atomic) magnetization directions $\left\{\hat{\mathbf{e}}_{M}^{\alpha}\right\}$ is not an extremum or a stationary solution of the total energy functional $E[n(\mathbf{r}), \mathbf{m}(\mathbf{r})]$. The constrained density functional theory developed by Dederichs et al. ${ }^{19}$ provides the necessary generalization to deal with arbitrary magnetic configurations-i.e., configurations where the orientations of the local moments are constrained to nonequilibrium directions. We define a generalized energy functional $\widetilde{E}\left[n(\mathbf{r}), \mathbf{m}(\mathbf{r}) \mid\left\{\hat{\mathbf{e}}_{M}^{\alpha}\right\}\right]$,

$\widetilde{E}\left[n(\mathbf{r}), \mathbf{m}(\mathbf{r}) \mid\left\{\hat{\mathbf{e}}_{M}^{\alpha}\right\}\right]$

$$
\begin{aligned}
& =E[n(\mathbf{r}), \mathbf{m}(\mathbf{r})]+\mu_{B} \sum_{\alpha} \mathbf{B}_{c}^{\alpha} \cdot\left(\left\langle\mathbf{m}^{\alpha}\right\rangle-\hat{\mathbf{e}}_{M}^{\alpha}\left\langle\hat{\mathbf{e}}_{M}^{\alpha} \mid \mathbf{m}^{\alpha}\right\rangle\right) \\
& =E[n(\mathbf{r}), \mathbf{m}(\mathbf{r})]+\mu_{B} \sum_{\alpha} \mathbf{B}_{c}^{\alpha} \cdot\left\{\mathbf{M}^{\alpha}-\mathbf{M}_{\|}^{\alpha}\right\} \\
& =E[n(\mathbf{r}), \mathbf{m}(\mathbf{r})]+\mu_{B} \sum_{\alpha} \mathbf{B}_{c}^{\alpha} \cdot \mathbf{M}_{\perp}^{\alpha}
\end{aligned}
$$

which consists of the energy functional of the unconstrained system $E[n(\mathbf{r}), \mathbf{m}(\mathbf{r})]$, extended by a constraint, which enforces that the direction $\mathbf{M}^{\alpha} / M^{\alpha}$ of the local (integrated) magnetic moment; i.e., the magnetization density averaged over the muffin-tin sphere of atom $\alpha,\left\langle\mathbf{m}^{\alpha}\right\rangle$,

$$
\left\langle\mathbf{m}^{\alpha}\right\rangle=\mathbf{M}^{\alpha}=\int_{M T^{\alpha}} \mathbf{m}(\mathbf{r}) d^{3} r,
$$

is parallel to the prescribed direction $\hat{\mathbf{e}}_{M}^{\alpha}$ and thus ensures that the local moments have no components $\mathbf{M}_{\perp}^{\alpha}$ normal to the directions $\hat{\mathbf{e}}_{M}^{\alpha}, \hat{\mathbf{e}}_{\perp}^{\alpha}$, for any atom. $\mathbf{M}_{\|}^{\alpha}$ is the component of $\mathbf{M}^{\alpha}$ parallel to $\hat{\mathbf{e}}_{M}^{\alpha} \cdot M_{\|}^{\alpha}$ is the projection of the local moment of the muffin-tin sphere $\alpha$ onto

$$
\left\langle\hat{\mathbf{e}}_{M}^{\alpha} \mid \mathbf{m}^{\alpha}\right\rangle=M_{\|}^{\alpha}=\hat{\mathbf{e}}_{M}^{\alpha} \cdot \mathbf{M}^{\alpha}=\hat{\mathbf{e}}_{M}^{\alpha} \cdot \int_{M T^{\alpha}} \mathbf{m}(\mathbf{r}) d^{3} r .
$$

Notice that for all sphere-averaged quantities only the spherical part of the magnetization density is needed, which makes the quantities easy to calculate. $\mathbf{B}_{c}^{\alpha}$ are Lagrange multipliers. Physically, they are transverse constraining fields acting in the direction $\hat{\mathbf{e}}_{\perp}^{\alpha}, \mathbf{B}_{c}^{\alpha}=\mathbf{B}_{\perp}^{\alpha}$. Minimizing Eq. (7) with respect to an electronic state yields the Kohn-Sham equations, which contains inside the muffin-tin sphere $\alpha$ an additional potential

$$
\mathbf{V}_{c}=\mu_{B} \boldsymbol{\sigma} \cdot \mathbf{B}_{\perp}^{\alpha},
$$

which is always perpendicular to $\hat{\mathbf{e}}_{M}^{\alpha}$. By means of the Hellmann-Feynman theorem the change $d E$ of the energy due to a directional change $d \hat{\mathbf{e}}_{M}^{\alpha}$ is given by the classical result

$$
d E=-\mu_{B} M_{\|}^{\alpha}\left(\hat{\mathbf{e}}_{M}^{\alpha}\right) \mathbf{B}_{c}^{\alpha}\left(\hat{\mathbf{e}}_{M}^{\alpha}\right) \cdot d \hat{\mathbf{e}}_{M}^{\alpha} .
$$

The difference vector $d \hat{\mathbf{e}}_{M}^{\alpha}$ is perpendicular to $\hat{\mathbf{e}}_{M}^{\alpha} \cdot{ }^{49}$

The effective $B$ field $\mathbf{B}_{e f f}^{\alpha}$ that enters the muffin-tin part of the Hamiltonian is given by (here, $\mathbf{B}_{e x t}$ is set to zero for simplicity)

$$
\begin{aligned}
\mathbf{B}_{e f f}^{\alpha}(\mathbf{r}) & =B_{x c}^{\alpha}\left[n(\mathbf{r}), m_{\|}(\mathbf{r})\right] \hat{\mathbf{e}}_{M}^{\alpha}+B_{\perp}^{\alpha} \hat{\mathbf{e}}_{\perp}^{\alpha} \\
& =B_{x c}^{\alpha}(\mathbf{r}) \hat{\mathbf{e}}_{M}^{\alpha}+B_{\perp}^{\alpha} \hat{\mathbf{e}}_{\perp}^{\alpha}=\mathbf{B}_{e f f}^{\alpha}(\mathbf{r}) \hat{\mathbf{e}}_{B}^{\alpha}(\mathbf{r}) .
\end{aligned}
$$

In order to calculate the exchange correlation $B$ field $\mathbf{B}_{x c}^{\alpha}(\mathbf{r})$ after the magnetization density is calculated from the wave functions, the magnetization density is projected onto the prescribed local quantization axis $\hat{\mathbf{e}}_{M}^{\alpha}$ and we obtain $m_{\|}^{\alpha}(\mathbf{r})$. Since the exchange correlation $B$ field is calculated from the projected magnetization density, the $B$ field is collinear. However, when a constraining field is added, the resulting effective $B$ field $\mathbf{B}_{e f f}^{\alpha}(\mathbf{r})$ is again a continuous noncollinear vector field in the muffin-tin spheres, with pointwise local directions $\hat{\mathbf{e}}_{B}^{\alpha}(\mathbf{r})$,

$$
\hat{\mathbf{e}}_{B}^{\alpha}(\mathbf{r})=\frac{B_{x c}^{\alpha}(\mathbf{r}) \hat{\mathbf{e}}_{M}^{\alpha}+B_{\perp}^{\alpha} \hat{\mathbf{e}}_{\perp}^{\alpha}}{\left\{\left[B_{x c}^{\alpha}(\mathbf{r})\right]^{2}+\left(B_{\perp}^{\alpha}\right)^{2}\right\}^{(1 / 2)}},
$$

different from the local quantization axis $\hat{\mathbf{e}}_{M}^{\alpha}$. Stocks and co-workers ${ }^{20,21}$ noticed this problem. They introduced an approximation that the constraint field $B_{c}^{\alpha}$ is an $\mathbf{r}$-dependent functional, rather than a constant, which has the same functional form as the exchange correlation $B$ field $B_{\perp}^{\alpha}$ $=c^{\alpha} \hat{\mathbf{e}}_{\perp}^{\alpha} B_{x c}^{\alpha}(\mathbf{r})$, where the scaling factor $c^{\alpha}$ replaces $B_{\perp}^{\alpha}$ as the parameter to be determined. The constraining $B$ fields $\mathbf{B}_{\perp}^{\alpha}$ that enter are often rather small and the approximation suggested by Stocks and co-workers might be a very good one, although we have not further investigated that. On the other hand, due to the introduction of the $\mathbf{r}$ dependence in the constraining fields, the approximation introduced a (possibly small) inconsistency between the constraining fields and the defining Eq. (7). A much stronger constraint condition than those discussed so far would be to demand that the magnetization density $\mathbf{m}(\mathbf{r})$ be parallel to $\hat{\mathbf{e}}_{M}^{\alpha}$ in every point inside a muffin-tin sphere. This constraint would result in a constraint 
field that depends on the position in the muffin-tin sphere. Maybe the approximation of Stocks and co-workers catches already this aspect.

In an actual constrained local moment (CLM) calculation $n(\mathbf{r}), \mathbf{m}(\mathbf{r})$, and $\mathbf{B}_{c}^{\alpha}$ have to be determined self-consistently. $n(\mathbf{r})$ and $\mathbf{m}(\mathbf{r})$ are calculated in the usual self-consistency cycle. At the same time the local constraint fields $\mathbf{B}_{c}^{\alpha}$ have to be adjusted, until the constraint condition $\left\langle\mathbf{m}^{\alpha}\right\rangle$ $-\hat{\mathbf{e}}_{M}^{\alpha}\left\langle\hat{\mathbf{e}}_{M}^{\alpha} \mid \mathbf{m}^{\alpha}\right\rangle=0$ is fulfilled. At the end of such a calculation we obtain the self-consistent densities and a set of local constraint $B$ fields that make the integrated magnetization perpendicular to the local spin quantization axes $\hat{\mathbf{e}}_{M}^{\alpha}$ vanish in each muffin-tin sphere. The total energy of the system is given by the constrained energy functional, Eq. (7). Since $\mathbf{B}_{c}^{\alpha}$ is always perpendicular to $\hat{\mathbf{e}}_{M}^{\alpha}$, the extra contribution to the total energy is $\int_{M T^{\alpha}} \mathbf{B}_{c}^{\alpha} \cdot \mathbf{m}(\mathbf{r}) d^{3} r$. However, the effective $B$ field that enters the Hamiltonian in the muffin-tin sphere of atom $\alpha$ is given by $\mathbf{B}_{e f f}^{\alpha}(\mathbf{r})=\mathbf{B}_{x c}^{\alpha}(\mathbf{r})+\mathbf{B}_{e x t}^{\alpha}(\mathbf{r})+\mathbf{B}_{c}^{\alpha}$. Therefore, the above contribution to the total energy cancels with the contribution of the constraint field to the kinetic energy. Thus, the constraint field does not enter the expression for the total energy explicitly, but it enters implicitly through the eigenvalues $\epsilon_{i}$ and through the self-consistent densities.

Demanding that the perpendicular component of the local moment $\mathbf{M}_{\perp}^{\alpha}$ vanish is not the only way to formulate a constraint to the direction of the local moment. There are several alternatives. A formulation that differs only formally from the constraint of Eq. (7) is to require that the cross product $\mathbf{M}^{\alpha} \times \hat{\mathbf{e}}_{M}^{\alpha}$ vanish. $\mathbf{M}^{\alpha} \times \hat{\mathbf{e}}_{M}^{\alpha}$ has the same magnitude as $\mathbf{M}_{\perp}^{\alpha}$ but is perpendicular to $\mathbf{M}_{\perp}^{\alpha}$. Using this constraint the additional term in the energy functional for each atom would be $\mathbf{B}_{c}^{\alpha} \cdot\left(\mathbf{M}^{\alpha} \times \hat{\mathbf{e}}_{M}^{\alpha}\right)$. Applying the Hellmann-Feynman theorem we find that the change of the energy is given by $d E$ $=-\mu_{B} \mathbf{M}^{\alpha} \cdot\left(\mathbf{B}_{c}^{\alpha} \times d \hat{\mathbf{e}}_{M}^{\alpha}\right)$. Therefore, the constraint field can be interpreted as a torque acting on the magnetic moment, in the spirit of the derivation of Antropov et al. ${ }^{15,16}$

The detailed formulation of constraints in the FLAPW method is given in Appendix A.2.

\section{Spin spirals}

A magnetic structure with moments $\mathbf{M}$ that are rotated by a constant angle from atom to atom along a certain direction of the crystal is called a spin spiral. This can be described by the propagation vector of the spin spiral $\mathbf{q}$, the rotation axis (which is, in the scalar-relativistic approximation, not fixed with respect to the lattice), and the relative angle $\vartheta$ between the magnetic moment and rotation axis. Upon translation by a lattice vector $\mathbf{R}$, the magnetic moment of an atom rotates by an angle $\varphi=\mathbf{q} \cdot \mathbf{R}$. Assuming a rotation around the $z$ axis (in the absence of spin-orbit coupling this is not a loss of generality), the magnetic moment of an atom $\alpha$ having the basis vector $\tau^{\alpha}$ in the unit cell $n$ (with the origin at the lattice vector $\mathbf{R}^{n}$ ) points in the direction

$$
\hat{\mathbf{e}}_{M}^{n \alpha}=\left(\begin{array}{c}
\cos \left(\mathbf{q} \cdot\left(\mathbf{R}^{n}+\boldsymbol{\tau}^{\alpha}\right)+\xi^{\alpha}\right) \sin \vartheta^{\alpha} \\
\sin \left(\mathbf{q} \cdot\left(\mathbf{R}^{n}+\boldsymbol{\tau}^{\alpha}\right)+\xi^{\alpha}\right) \sin \vartheta^{\alpha} \\
\cos \vartheta^{\alpha}
\end{array}\right) .
$$

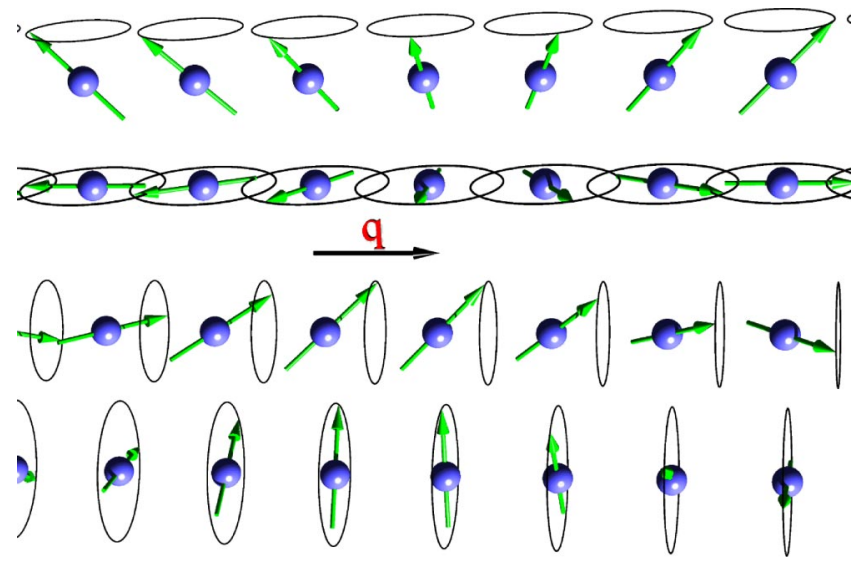

FIG. 1. (Color online). Four examples of spin-spirals with spinrotation axis perpendicular (upper two) and parallel (lower two) to the spin-spiral vector q. For each case two spirals with angles of $\vartheta=\pi / 2$ and $\vartheta=\pi / 4$ between the magnetic moment and rotation axes are shown.

For more than one magnetic atom in the unit cell, in addition to its basis vector $\tau_{\alpha}$, an additional atom-dependent phase $\xi^{\alpha}$ appears in the above equation. Nevertheless, the magnetic moments of all atoms have to rotate around the same axis. To distinguish from the longitudinal spin-density waves, spin spirals are also frequently called helical magnetic structures, spiral spin-density waves, or frozen magnons. The origin of the last term is that a spin spiral looks like a "snapshot" of a single magnon at a fixed time. Spin-spiral calculations can therefore be used to simulate the effect of temperature on a magnetic system in the adiabatic approximation, in particular at very low temperatures, when magnons with long wavelength dominate. Another possible application of spin spirals is the simulation of domain walls including the calculation of the formation energy. Without the application of the generalized Bloch theorem the investigation of such magnetic structures requires very large unit cells. Since the spin spiral is an exact solution of the classical Heisenberg model at $T=0$, it is believed that they cover a large and important part of the phase space of possible spin states. Thus among all possible magnetic states, spin spirals are the next relevant class of spin states besides the high-symmetry magnetic states-i.e., the ferromagnetic, antiferromagnetic, or ferromagnetic configurations. Though there are many possible applications for spin-spiral calculations, it was the discovery of a spiral ground-state structure in fcc iron ${ }^{17}$ and $4 f$ metals $^{22}$ that gave rise to many theoretical studies. ${ }^{5,23}$

Figure 1 shows four examples of spin spirals with spinrotation axis perpendicular (upper two) and parallel (lower two) to the spin-spiral vector $\mathbf{q}$ and different angles between the spin-rotation axis and magnetic moments. When spinorbit coupling is neglected, the two spirals with the same $\vartheta$ in Fig. 1 become completely equivalent. However, the spin spirals with different $\vartheta$ do not become equivalent. $\vartheta$ is still a well-defined quantity, if SOC is neglected, because the rotation axis is a vector (direction) in spin space. For systems with one atom per unit cell the angle $\vartheta$ may be determined by higher-order spin interactions-i.e., interactions beyond the Heisenberg model. For unit cells with more than one 
magnetic atom, conical spin structures $(\vartheta \neq \pi / 2)$ can be a consequence of competitive exchange interactions between the different atoms. The total energy $E\left(\mathbf{q},\left\{\vartheta^{\alpha}\right\},\left\{\xi^{\alpha}\right\}\right)$ depends on the wave vector of the spin spiral, the cone angles, and the relative phases between the atoms in the unit cell. Therefore, the investigation of the minimum of the total energy-i.e., the magnetic ground state-is a search with many degrees of freedom. To study $E$ as a function of $\vartheta^{\alpha}$ or $\xi^{\alpha}$ further requires a calculation with constraint fields.

A very elegant treatment of spin spirals by first-principles calculations is possible when the generalized Bloch theorem ${ }^{24,25}$ is applied. However, this theorem can only be proved when SOC is neglected. For this reason the spinrotation axis will always be considered as parallel to the $z$ axis of the spin coordinate frame. Thus, only the $m_{x}$ and $m_{y}$ components are rotated, while $m_{z}$ does not change. Following Sandratskii ${ }^{25}$ we can define a generalized translation $\mathcal{T}_{n}$ that combines a lattice translation and a spin rotation. Applying a generalized translation to $\mathcal{H} \psi$ yields

$$
\begin{aligned}
\mathcal{T}_{n} \mathcal{H}(\mathbf{r}) \boldsymbol{\psi}(\mathbf{r})= & \mathbf{U}\left(-\mathbf{q} \mathbf{R}^{n}\right) \mathcal{H}\left(\mathbf{r}+\mathbf{R}^{n}\right) \mathbf{U}^{\dagger}\left(-\mathbf{q} \mathbf{R}^{n}\right) \mathbf{U}\left(-\mathbf{q} \mathbf{R}^{n}\right) \\
& \times \boldsymbol{\psi}\left(\mathbf{r}+\mathbf{R}^{n}\right)=\mathcal{H}(\mathbf{r}) \mathbf{U}\left(-\mathbf{q} \mathbf{R}^{n}\right) \boldsymbol{\psi}\left(\mathbf{r}+\mathbf{R}^{n}\right),
\end{aligned}
$$

where $\mathbf{U}\left(\mathbf{q} \mathbf{R}^{n}\right)$ is the spin-1/2 rotation matrix:

$$
\mathbf{U}\left(\mathbf{q} \mathbf{R}^{n}\right)=\left(\begin{array}{cc}
e^{-i \varphi / 2} & 0 \\
0 & e^{i \varphi / 2}
\end{array}\right), \quad \varphi=\mathbf{q} \cdot \mathbf{R}^{n}
$$

In analogy with the proof of Bloch's theorem ${ }^{26}$ it follows that the eigenstates can be chosen such that

$$
\mathcal{T}_{n} \psi(\mathbf{k}, \mathbf{r})=\mathbf{U}\left(-\mathbf{q} \mathbf{R}^{n}\right) \psi\left(\mathbf{k}, \mathbf{r}+\mathbf{R}^{n}\right)=e^{i \mathbf{k} \cdot \mathbf{R}^{n}} \psi(\mathbf{k}, \mathbf{r}) .
$$

This formulation of the generalized Bloch theorem is equivalent to the statement that the eigenstates of the Hamiltonian can be written in the form ${ }^{11}$

$$
\boldsymbol{\psi}(\mathbf{k}, \mathbf{r})=e^{i \mathbf{k} \cdot \mathbf{r}}\left(\begin{array}{c}
e^{-i \frac{\mathbf{q}}{2} \cdot \mathbf{r}} F(\mathbf{k}, \mathbf{r}) \\
e^{+i \frac{\mathbf{q}}{2} \cdot \mathbf{r}} G(\mathbf{k}, \mathbf{r})
\end{array}\right)
$$

where $F(\mathbf{k}, \mathbf{r})$ and $G(\mathbf{k}, \mathbf{r})$ are functions with translational periodicity-e.g., $F\left(\mathbf{k}, \mathbf{r}+\mathbf{R}^{n}\right)=F(\mathbf{k}, \mathbf{r})$. We will give the formulas that arise in the implementation of spin spirals in the FLAPW method in Appendix A.3.

\section{APPLICATIONS}

In this section we show examples of the calculation of the noncollinear magnetism on two systems: an unsupported hexagonal monolayer of $\mathrm{Cr}$ and bulk $\mathrm{Fe}$ in both a bcc and a fcc structure. The concepts of Sec. II were implemented in the FLEUR code, a realization of the FLAPW method ${ }^{27,28}$ suitable for bulk and film systems.

\section{A. Unsupported $\mathrm{Cr}$ monolayers}

To check our implementation of noncollinear magnetism, we performed tests for an unsupported monolayer (UML) of $\mathrm{Cr}$ with the symmetry and the lattice constant of a monolayer on the $\operatorname{Ag}(111)$ surface. We chose the theoretical LDA Ag lattice constant of 7.79 a.u. The k-point set that we employed corresponds to 180 points in the full two-dimensional Brillouin zone, the plane-wave cutoff was set to $K_{\max }$ $=3.3$ a.u. $^{-1}$ leading to a basis set with about 130 basis function per atom, and the muffin-tin radius was chosen as large as possible, $R_{M T}=2.75$ a.u. We applied the LDA parametrization according to Moruzzi, Janak, and Williams. ${ }^{29}$

\section{Intra-atomic noncollinearity}

In Sec. II A we described our way of implementing noncollinear magnetism with a "hybrid" approach that uses a continuous vector magnetic field in the interstitial and vacuum region that smoothly connects the collinear intraatomic $B$ fields inside the muffin-tin spheres. While the potential matrix $\mathbf{V}$ has-in the local frame of a muffin-tin sphere-no off-diagonal elements, the density matrix $\boldsymbol{\rho}$ is generally nondiagonal in spin space and, therefore, still describes a continuous intra-atomic noncollinear magnetization density. Since the basis functions inside a muffin-tin sphere are coupled to the plane waves of the interstitial region, the influence of the $\mathbf{B}$ field outside influences the charge and magnetization density inside a muffin-tin sphere. This can now be used to estimate the intra-atomic noncollinearity and helps-if there is any-to adjust the size of the muffin-tin spheres so that all noncollinear effects are well outside the spheres.

We calculated an hexagonal unsupported monolayer of $\mathrm{Cr}$ for the in-plane lattice constant of the $\operatorname{Ag}(111)$ surface. The magnetic state was chosen to be the $120^{\circ}$ Néel state, where all magnetic moments have a relative angle of $120^{\circ}$ and the unit cell is the $\sqrt{3} \times \sqrt{3} R 30^{\circ}$ unit cell containing three atoms. This state is the solution of the classical Heisenberg model for antiferromagnets in nearest-neighbor approximation. In our calculations this state also had the lowest total energy compared to all states that are allowed as solutions of the Heisenberg model in the next-nearest-neighbor approximation. ${ }^{30}$ From Fig. 2 we see that in a sphere centered around the nucleus up to a radius of 2 a.u. the magnetization density remains fairly collinear. In the usual calculations we use muffin-tin radii of 2.1-2.3 a.u.; therefore the approximation of a collinear description within the muffintin sphere can be expected to be a good one for these systems. Hobbs and Hafner ${ }^{31}$ found a similar behavior for $\mathrm{Cr}$ on $\mathrm{Cu}(111)$ with a full vector-magnetization density description. Moreover, using different muffin-tin radii we have the possibility to check the quality of our approximation as will be shown in Sec. III B 5.

For (magnetic) high-symmetry states (e.g., in this case the Néel state or spin spirals with $\vartheta=\pi / 2$ and all collinear states) the average direction of the magnetization within a muffin-tin sphere $\alpha,\left\langle\mathbf{m}^{\alpha}\right\rangle$, will be in line with the direction of the $B$ field, $\hat{\mathbf{e}}^{\alpha}$, and the magnetic state denotes a stationary state to the solution of the Kohn-Sham equations. But gen- 


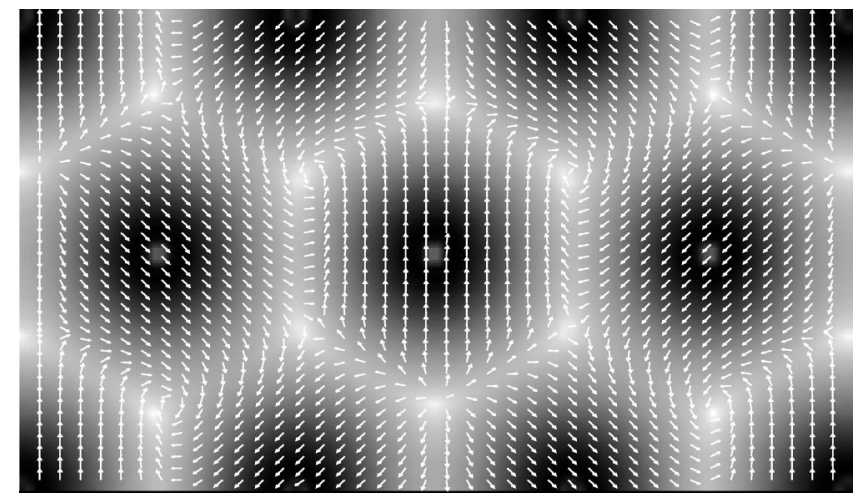

FIG. 2. Fully noncollinear output magnetization density of a UML of $\mathrm{Cr}$ in the $120^{\circ}$ Néel state. Light (dark) areas indicate regions of low (high) magnetization; arrows indicate the direction of the magnetization density. The $\mathrm{Cr}$ atoms are located in the center of the dark hexagons and are surrounded by touching muffin-tin spheres.

erally, if the directions $\hat{\mathbf{e}}^{\alpha}$ do not specify such a highsymmetry state, $\left\langle\mathbf{m}^{\alpha}\right\rangle$ will be no longer in line with $\hat{\mathbf{e}}^{\alpha}$ and this effect can be used to "relax" the directions of the magnetic moments as will be shown in Section III B 1.

\section{Cr: Constraint}

As a first test of the implementation of the constrained local moment method we compared the change of the total energy for small constraining $B$ fields with the energy calculated in first-order perturbation theory. We performed nonself-consistent calculations, setting the constraint field to different values manually, rather than calculating it selfconsistently. In other words, we applied small perpendicular $B$ fields inside each muffin-tin sphere, using the implementation for constrained local moment calculations. We tried fields with different magnitudes, which were smaller than the constraint field necessary to make the perpendicular moment in the respective muffin-tin sphere vanish. Such fields represent a small perturbation of the system. In first-order perturbation theory the energy difference between the unperturbed and perturbed systems is given by the sum over the expectation values of all occupied states of the unperturbed system with the perturbation term of the Hamiltonian. If we write the contribution to the Hamiltonian due to the perpendicular field within the local spin coordinate frame as

$$
\begin{gathered}
\mathcal{H}_{M T^{\alpha}}^{\text {off-d }}=-\mu_{B} \delta_{\sigma^{\alpha},-\sigma^{\alpha}}\left[B_{c, x}^{\alpha}+\operatorname{sgn}\left(\sigma^{\alpha}\right) i B_{c, y}^{\alpha}\right], \\
\text { with } \operatorname{sgn}\left(\sigma^{\alpha}\right)=\left\{\begin{array}{rc}
1 & \text { for } \sigma^{\alpha}=\uparrow, \\
-1 & \text { for } \sigma^{\alpha}=\downarrow,
\end{array}\right.
\end{gathered}
$$

we find that the energy difference is given by

$$
\begin{aligned}
\Delta E & =-\mu_{B} \sum_{\nu \alpha} \int_{M T^{\alpha}} \boldsymbol{\psi}_{\nu}^{*}(\mathbf{r})\left(\boldsymbol{\sigma}_{x} B_{x}^{\alpha}+\boldsymbol{\sigma}_{y} B_{y}^{\alpha}\right) \boldsymbol{\psi}_{\nu}(\mathbf{r}) d^{3} r \\
& =-\mu_{B} \sum_{\alpha}\left(M_{x}^{\alpha} B_{x}^{\alpha}+M_{y}^{\alpha} B_{y}^{\alpha}\right),
\end{aligned}
$$

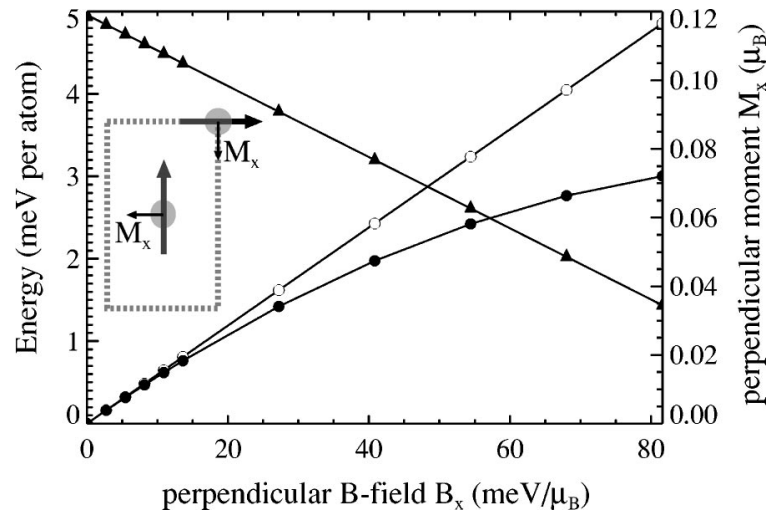

FIG. 3. The total energy difference (solid circles) and the product $-\mu_{B} M_{x}^{\alpha} B_{x}^{\alpha}$ (open circles) is plotted as a function of the applied perpendicular $B$ field $\left(B_{x}^{\alpha}\right)$. The triangles show the perpendicular local magnetic moment $\left(M_{x}^{\alpha}\right)$.

where $M_{(x, y)}^{\alpha}$ and $B_{(x, y)}^{\alpha}$ are the respective components of the local output magnetization and the input $\mathbf{B}$ field within the sphere $\alpha$ in the local spin coordinate frame. Note that also, in Eq. (19), $\sigma^{\alpha}$ is the spin of atom $\alpha$ in its local frame of reference and is, therefore, either purely spin up $(\uparrow)$ or spin down $(\downarrow)$.

In a test calculation, we used a unit cell containing two atoms according to the inset of Fig. 3. The magnetic moments of the two atoms are aligned with a relative angle of $90^{\circ}$, so that the configuration consists of alternating rows of atoms with moments pointing, e.g, in the $y$ and $x$ directions, respectively.

From a self-consistent calculation without a constraint field we obtained small perpendicular moments of about $0.12 \mu_{B}$ inside the muffin-tin spheres. The direction of the perpendicular moments is shown in the inset of Fig. 3, indicating the tendency of the system to evolve into a state of alternating antiferromagnetic rows. The local spin coordinate frame of each atom was chosen such that the perpendicular moment points along the $x$ axis of the local frame. Starting from the self-consistent charge and magnetization densities we performed non-self-consistent calculations where we applied a perpendicular field also in the $x$ direction and with the same magnitude in each muffin-tin sphere. Figure 3 shows the change of the energy (i.e., sum of the single-particle energies) as a function of the applied perpendicular $B$ field (solid circles). For very small fields this energy difference is in excellent agreement with the energy difference calculated in first-order perturbation theory (open circles) according to Eq. (20). But already for fields as small as $20 \mathrm{meV} / \mu_{B}$ (for comparison, the average exchange correlation $B$ field is about $2500 \mathrm{meV} / \mu_{B}$ inside the muffin tins) the result of the perturbation theory starts to deviate from the calculated energy. In first-order perturbation theory the effect of the change of the eigenfunctions due to the perturbation is neglected. As a measure for that change we can take the output perpendicular moment of the calculation, which is also shown in Fig. 3 (solid triangles). It can be seen that $M_{x}^{\alpha}$ decreases linearly with increasing field. At a field of about $60 \mathrm{meV} / \mu_{B}$ it has already decreased to half its original size. 
Hence, the first-order perturbation theory is only accurate for very small $B$ fields below $20 \mathrm{meV} / \mu_{B}$.

As we have pointed out in Sec. II B, the local constraint fields $\mathbf{B}_{c}^{\alpha}$ have to be determined self-consistently-e.g., using an iteration scheme. Thus, after each iteration or set of iterations that converges $n(\mathbf{r})$ and $\mathbf{m}(\mathbf{r})$, a correction $\Delta \mathbf{B}_{c}^{\alpha}$ to the current estimate $\mathbf{B}_{c, \text { in }}^{\alpha}$ needs to be determined:

$$
\mathbf{B}_{c, \text { out }}^{\alpha}=\mathbf{B}_{c, \text { in }}^{\alpha}+\Delta \mathbf{B}_{c}^{\alpha} .
$$

Naturally, we expect $\Delta \mathbf{B}_{c}^{\alpha}$ to be proportional to the perpendicular magnetic moment in the sphere, $\mathbf{M}_{\perp}^{\alpha}=\int_{M T^{\alpha}}\{\mathbf{m}(\mathbf{r})$ $\left.-\hat{\mathbf{e}}^{\alpha}\left[\hat{\mathbf{e}}^{\alpha} \cdot \mathbf{m}(\mathbf{r})\right]\right\} d^{3} r$. The proportionality factor should be chosen such that $\mathbf{B}_{c}^{\alpha}$ approaches the self-consistent value quickly, but does not overshoot. In principle, it would be best to know $d \mathbf{M}_{\perp}^{\alpha} / d \mathbf{B}_{c}^{\alpha}$, but this quantity is not easily accessible. However, if we assume that rotating $\mathbf{B}_{\text {eff }}^{\alpha}$ by some angle rotates $\mathbf{M}^{\alpha}$ by the same angle, we arrive at the following choice for the correction to the constraint field:

$$
\Delta \mathbf{B}_{c}^{\alpha}=-\left|\left\langle\mathbf{B}_{e f f}^{\alpha}\right\rangle\right| \frac{\mathbf{M}_{\perp}^{\alpha}}{\left|\mathbf{M}^{\alpha}\right|},
$$

where $\mathbf{M}^{\alpha}=\int_{M T^{\alpha}} \mathbf{m}(\mathbf{r}) d^{3} r$ is the integrated magnetic moment in the muffin tin and $\left\langle\mathbf{B}_{e f f}^{\alpha}\right\rangle$ is the average effective $B$ field in the sphere. All quantities on the right-hand side are output of a self-consistency iteration. To generalize this formula and to improve the convergence we can add a scaling factor $\beta_{c}$ :

$$
\Delta \mathbf{B}_{c}^{\alpha}=-\beta_{c}\left|\left\langle\mathbf{B}_{e f f}^{\alpha}\right\rangle\right| \frac{\mathbf{M}_{\perp}^{\alpha}}{\left|\mathbf{M}^{\alpha}\right|} .
$$

Our test calculations show that for systems where the size of the local moment $\left|\mathbf{M}^{\alpha}\right|$ does not change much with $\mathbf{B}_{c}^{\alpha}$, the constraint field and densities can be converged simultaneously, and the linear mixing of the former does not interfere with the Broyden mixing scheme applied to the latter.

As a second test we compared the calculated total energy $[\widetilde{E}$, according to Eq. (7)] to the energy obtained from the constraint $B$ fields using the Hellmann-Feynman theorem expressed in Eq. (11). Equation (11) can be used to calculate the energy difference between two magnetic states by an integration over a path of magnetic configurations that connects the two states. This method has been used by Oswald et $a l .{ }^{32}$ to calculate the energy difference between a ferromagnetic and an antiferromagnetic state of $3 d$-impurity dimers in $\mathrm{Cu}, \mathrm{Ag}$, and $\mathrm{Pd}$. We chose again the UML $\mathrm{Cr} /$ $\operatorname{Ag}(111)$ system as in the previous paragraphs, using the same computational parameters. As starting and final (magnetic) states we consider the ferromagnetic and a row-wise antiferromagnetic configuration. Rotating one of the two atoms in the unit cell, as illustrated in the inset of Fig. 4, yields a path of magnetic states connecting the initial and final states. This path is described by a single parameter, the angle $\varphi$. If $\varphi$ is changed by an infinitesimal step $d \varphi$, the change of the direction of the local moment $d \hat{\mathbf{e}}$ is always parallel to the local constraint field $\mathbf{B}_{c}^{\varphi}$ in such a configuration. Now the

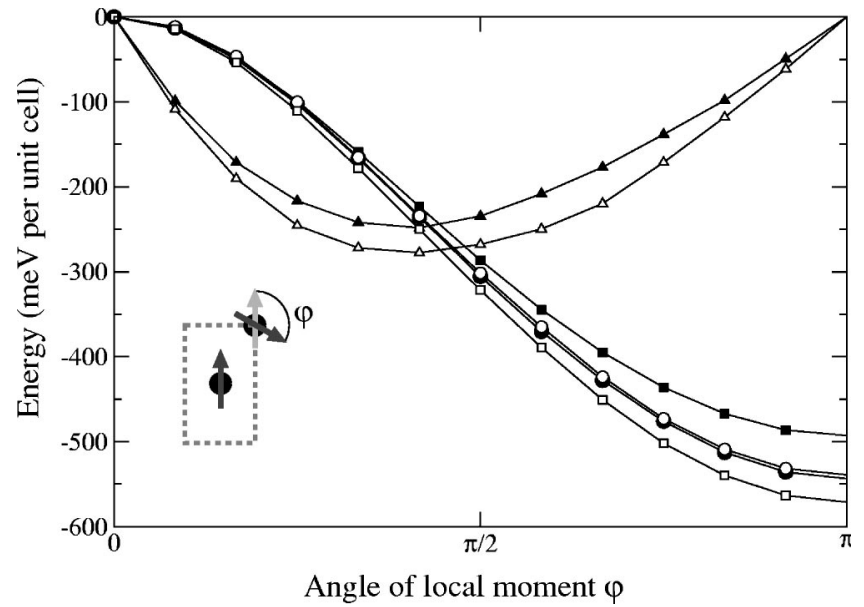

FIG. 4. The total energy of an UML $\mathrm{Cr}(111)$ with the lattice constant of $\operatorname{Ag}(111)$ as a function of the relative angle $\varphi$ of the local moment. The plot contains the results of two calculations with different muffin-tin radii of $R_{M T}=2.75$ a.u. (solid symbols) and $R_{M T}$ $=2.0$ a.u. (open symbols). Shown are the calculated total energy relative to the ferromagnetic energy (circles) and the product of the local moment and constraint $B$ field, $-\mu_{B} M_{\|}(\varphi) B_{c}(\varphi)$ (triangles) and $-\mu_{B} \int M_{\|}(\varphi) B_{c}(\varphi) d \varphi$ (squares).

Hellmann-Feynman theorem, Eq. (11), can be integrated, yielding an equation for the energy difference between the ferromagnetic state and a state with angle $\varphi$ between the magnetic moments:

$$
E(\varphi)-E(\varphi=0)=-\mu_{B} \int_{0}^{\varphi} M_{\|}(\varphi) B_{c}(\varphi) d \varphi
$$

The result of the test calculation is shown in Fig. 4. We first concentrate on the results obtained for a muffin-tin radius of 2.75 a.u., denoted by the solid symbols. The graph shows the calculated total energy $(\widetilde{E})$ difference (solid circles), the product $-\mu_{B} M_{\|}(\varphi) B_{c}(\varphi)$ (solid triangles), and the integral of the latter quantity (solid squares). The energy decreases with increasing angle. It shows a cosinelike behavior as expected from the nearest-neighbor Heisenberg model for an antiferromagnetic material. The shape of the $-\mu_{B} M_{\|}(\varphi) B_{c}(\varphi)$ curve is dominated by $B_{c}(\varphi) . M_{\|}(\varphi)$ is almost constant. It changes only within a range of $4.1 \mu_{B}-4.2 \mu_{B} . M_{\|}(\varphi)$ also changes very little (less than $1 \%$ ) from a calculation with the constraint field switched off to a constraint calculation. The symmetric magnetic statesferromagnetic and antiferromagnetic - represent extrema of the total energy. Hence, they are stable magnetic solutions. Consequently, the constraint field-and thus $-\mu_{B} M_{\|}(\varphi) B_{c}(\varphi)$-is zero for $\varphi=0$ and $\varphi=\pi$. $-\mu_{B} M_{\|}(\varphi) B_{c}(\varphi)$ is a continuous function that reaches a maximum at an intermediate angle slightly smaller than $\pi / 2$.

The integral $-\mu_{B} \int M_{\|}(\varphi) B_{c}(\varphi) d \varphi$ exhibits the same behavior as the total energy, but has a slightly smaller magnitude than the calculated total energy difference. At the final (antiferromagnetic) state the two curves differ by about $9 \%$. We suspect that this might be due to incomplete-basis-set corrections. The Hellmann-Feynman theorem is based on the 
fact that the functional derivative of the total energy with respect to the (components of the) density (matrix) vanishes, $\delta E / \delta \boldsymbol{\rho}=0$, because $\boldsymbol{\rho}$ is already the ground-state density and minimizes $E$. Therefore, only the explicit dependence of the energy on an external parameter has to be taken into account when differentiating the energy with respect to the parameter. In our case this parameter is the direction of the local moment. However, in methods like the FLAPW method the basis set depends on the external parameters. The corresponding corrections are called the incomplete-basis-set corrections. Such corrections play an important role in the calculation of the atomic forces. ${ }^{33}$ In fact, in atomic force calculations the incomplete-basis-set corrections are usually even larger than the Hellmann-Feynman force. Recently, Grotheer and Fähnle have derived an expression for the incomplete-basis-set corrections to the torque acting on the localized magnetic moments. ${ }^{34}$ They have performed calculations similar to that presented in Fig. 4 on bcc Fe and found that the corrections are about $2 \%$ of the size of the Hellmann-Feynman torque.

The basis function of the FLAPW method in the interstitial region are plane waves. Thus, the basis set is independent of the local quantization axis $\hat{\mathbf{e}}^{\alpha}$ in that region. Hence, changing the muffin-tin radii $R_{M T}^{\alpha}$ should have an effect on the size the incomplete-basis-set corrections. To gain an indication whether the incomplete-basis-set corrections are the cause of the difference between the calculated total energy difference and the energy difference obtained from the HellmannFeynman theorem, we have repeated the calculation with a much smaller muffin-tin radius of $R_{M T}=2.0$ a.u. instead of 2.7 a.u. With this choice the volume covered by the muffintin spheres is reduced by more than $60 \%$. The result of this second calculation is also shown in Fig. 4 by the open symbols. The product $-\mu_{B} M_{\|}(\varphi) B_{c}(\varphi)$ is changed considerably. The energy difference calculated from the HellmannFeynman theorem is now about $6 \%$ too large; i.e., the deviation has changed sign and its magnitude has become smaller. This result supports the idea that the deviation is due to the incomplete-basis-set corrections. However, to definitely settle this point, calculations including these corrections would be necessary. Such calculations remain a future project.

\section{B. bulk Fe}

Stocks and co-workers ${ }^{20,21}$ chose bcc Fe to test their implementation of constrained local moment calculations. Also Grotheer and Fähnle ${ }^{34}$ used the same test system for their implementation of the incomplete-basis-set corrections to the Hellmann-Feynman torque on the magnetic moments. Therefore, we repeated the test for bcc Fe using the same geometry as Stocks and co-workers. In particular, we used the same LDA lattice constant of $a_{0}=5.27$ a.u. Our $k$-point set corresponds to $2016 k$ points in the full three-dimensional Brillouin zone. The plane-wave cutoff was set to $K_{\max }$ $=4.0$ a.u. $^{-1}$ leading to a basis set with about 80 basis functions per atom. We chose a muffin-tin radius of $R_{M T}$ $=2.25$ a.u. fcc or $\gamma$-Fe has been extensively studied for many years. One of the reasons for the large experimental and theoretical attention this system has attracted is the Invar properties of alloys based on $\gamma$-Fe. This interest has been renewed by neutron diffraction experiments by Tsunoda, ${ }^{17}$ who found that the ground state of $\gamma$-Fe most likely consist of a noncollinear, spiral magnetic structure. This discovery stimulated many noncollinear $a b$ initio investigations by different authors. ${ }^{5,11,23,35-41}$ The large amount of ab initio data on $\gamma$-Fe spin spirals make this an ideal test system for a spinspiral implementation. Here we have used the experimental $\mathrm{Cu}$ lattice constant of $a=6.82$ a.u., since the $\gamma$-Fe has been found for $\mathrm{Fe}$ in a $\mathrm{Cu}$ matrix. A tetragonal unit cell containing two atoms was used. In Sec. III B 3 a $k$-point set corresponding to $1120 \mathbf{k}$ points in the full three-dimensional Brillouin zone (BZ) and two different plane-wave cutoffs $K_{\max }$ $=4.0$ a.u $^{-1}$ and 4.4 a.u. $^{-1}$ corresponding to 85 and 115 basis functions per atom, respectively, were used. The calculations of Secs. III B 4 and III B 5 were performed with $k$-point sets corresponding to $5632 \mathbf{k}$ points in the full BZ and plane-wave cutoffs of $K_{\max }=4.2$ a.u. $^{-1}, 4.6$ a.u. $^{-1}$, and 5.0 a.u. $^{-1}$ for muffin-tin radii of 1.9 a.u., 2.1 a.u., and 2.3 a.u., respectively.

\section{Relaxation of the magnetic moments}

As we described in Sec. III A 1, the output magnetization density obtained from solving the Kohn-Sham equations is in general noncollinear inside the muffin-tin spheres. Of course, in our method this information about intra-atomic noncollinearity is lost again after the construction of the potential matrix that assumes that $B_{x c}^{\alpha}$ is collinear inside the muffin-tin spheres and points in the direction $\hat{\mathbf{e}}^{\alpha}$. In the self-consistency cycle we can either "constrain" the direction of the magnetic moments so that the average direction of the magnetization, $\left\langle\mathbf{m}^{\alpha}\right\rangle$, is parallel to $\hat{\mathbf{e}}^{\alpha}$ or "relax" the direction of the magnetic moments by adjusting $\hat{\mathbf{e}}^{\alpha}$ in the direction of $\left\langle\mathbf{m}^{\alpha}\right\rangle$ until $\hat{\mathbf{e}}^{\alpha}||\left\langle\mathbf{m}^{\alpha}\right\rangle$. Using the latter procedure, we have a tool to determine the magnetic ground state or a local metastable state of a system within a chosen unit cell.

In such a calculation we also apply the approximation of a collinear magnetization density inside each muffin-tin sphere, $\mathbf{m}(\mathbf{r})=m^{\alpha}(\mathbf{r}) \hat{\mathbf{e}}_{M}^{\alpha}$; only the directions $\hat{\mathbf{e}}_{M}^{\alpha}$ [and consequently $\left.m^{\alpha}(\mathbf{r})\right]$ are relaxed. In order to relax the magnetic configuration it is necessary to calculate the total (integrated) perpendicular output magnetization

$$
\left\langle\mathbf{m}_{\perp, \text { out }}^{\alpha}\right\rangle=\mathbf{M}_{\perp, \text { out }}^{\alpha}=\int_{M T_{\alpha}} \mathbf{m}_{\perp, \text { out }}^{\alpha}(\mathbf{r}) d^{3} r
$$

in addition to $\mathbf{M}_{\| \text {,out }}^{\alpha}$. The output direction $\hat{\mathbf{e}}_{\text {out }}^{\alpha,(i)}=\left(\mathbf{M}_{\perp, \text { out }}^{\alpha}\right.$ $\left.+\mathbf{M}_{\|, \text {out }}^{\alpha}\right) /\left|\mathbf{M}_{\perp, \text { out }}^{\alpha}+\mathbf{M}_{\|, \text {out }}^{\alpha}\right|$ at each site $\alpha$ differs normally from the input direction (the orientation of the spins at the beginning of the iteration step $i) \hat{\mathbf{e}}_{i n}^{\alpha,(i)}$. For the next iteration $i+1$, the input direction $\hat{\mathbf{e}}_{i n}^{\alpha,(i+1)}$ is changed independently from the charge density and the size of the magnetizations, $\left|\mathbf{M}_{\perp, \text { out }}^{\alpha}\right|$ or $\left|\mathbf{M}_{\|, \text {out }}^{\alpha}\right|$. In our implementation the parameters describing the orientation are the azimuthal and polar angles 


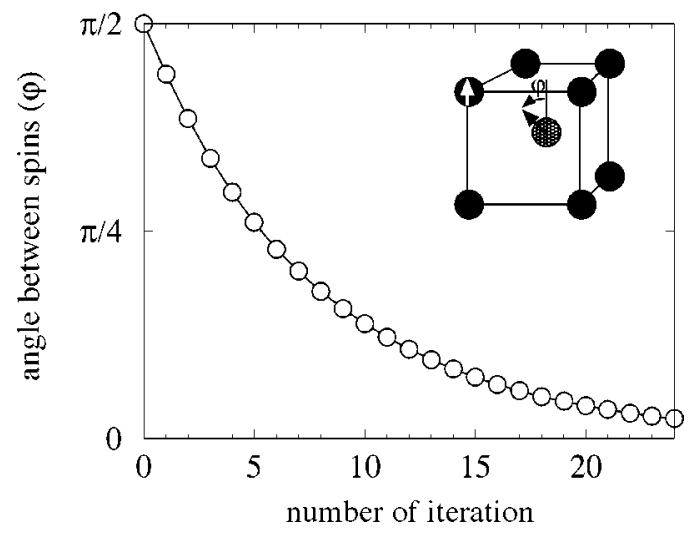

FIG. 5. Relaxation of the spin direction of a Fe atom in bulk bcc Fe. The evolution of the angle $\varphi$ between the magnetic moments of two $\mathrm{Fe}$ atoms is shown as a function of the number of the selfconsistency steps.

$\varphi$ and $\theta$, respectively. We used straight mixing to determine the input orientation for the next iteration step: For example, the angle $\varphi_{i n}^{\alpha,(i+1)}$ being used in the next iteration is chosen as

$$
\varphi_{\text {in }}^{\alpha,(i+1)}=(1-\beta) \varphi_{\text {in }}^{\alpha,(i)}+\beta \varphi_{\text {out }}^{\alpha,(i)},
$$

where $\beta$ is a mixing parameter. The choice of the mixing parameter for the angles crucially determines the speed of convergence and has to be adapted to a specific system. Convergence of the charge and magnetization density and the directions can be done simultaneously; i.e., after each selfconsistency iteration of the densities a new set of angles is determined. The mixing scheme and the mixing parameters are chosen independently for the densities and directions. However, when a Broyden mixing scheme is used for the densities, the Broyden "memory" has to be deleted regularly (every 10 iterations) while the directions are still changing quickly.

As an example we calculated the relaxation of the angle $\varphi$ between the magnetic moments of two Fe atoms in the bcc unit cell starting from a canted spin structure $\left(\varphi=90^{\circ}\right)$. As sketched in Fig. 5 the spin directions of the corner atoms are kept fixed. The self-consistent determination of the relative ground-state angles between these two atoms was started using a converged charge density for this particular relative starting angle. Then the variation of the orientation is carried out as described above. We used $\beta=1.0$, which means that the mixing is $100 \%$ for the directions of local the moments. The evolution of the angle $\varphi$ with the number of selfconsistency steps is shown in Fig. 5. As we expect, $\varphi$ converges towards $0^{\circ}$ to yield the ferromagnetic solution. Selfconsistency of the densities and directions is obtained in about 25 iterations.

For Co, not shown here, we overrelaxed the directions of the moments by using $\beta=3.0$, which means that the mixing is even more than $100 \%$ for the directions of the local moments. The convergence of the orientation is very fast and the ferromagnetic state is reached after only 5 iterations. Due to the fast change of the directions, the charge $n(\mathbf{r})$ and magnetization density $m_{(\|)}(\mathbf{r})$ are not converged anymore and a further 15 iterations are needed basically to converge those quantities. After 20 iterations both the densities and directions are converged. This shows that by separating the orientational degrees of freedom of the magnetic moment from the charge density and the size of the magnetization during the iteration progress, the convergence of the orientation of spins can be accelerated considerably.

\section{2. bcc Fe: Constraint}

To compare with the constraint results of Stocks and coworkers we started with an unconstrained calculation of bcc Fe. In such a calculation the magnetization density is projected onto the local quantization axis $\hat{\mathbf{e}}^{\alpha}$ inside the muffin tins after each iteration; i.e., the perpendicular magnetic moment $\mathbf{M}_{\perp}^{\alpha}$ is neglected. Although these calculations can be converged to a stable solution, the result is not self-consistent in the sense that the direction of the output magnetic moment is not equal the direction of the input moment, $\hat{\mathbf{e}}_{\text {out }}^{\alpha} \neq \hat{\mathbf{e}}_{\text {in }}^{\alpha}$ $=\hat{\mathbf{e}}^{\alpha}$.

The results of the bcc Fe calculations are presented in Fig. 6. Panel (b) contains the parallel magnetic moment $M_{\|}(\varphi)$ (open diamonds) and the perpendicular (output) moment $M_{\perp}(\varphi)$ (open triangles). Our results are not in agreement with those of Stocks and co-workers. In particular, we find that the moment decreases strongly, by more than $50 \%$, when it is rotated towards the antiferromagnetic state. Stocks and co-workers obtain a very similar magnetic moment for the ferromagnetic state. However, in their study the moment varies only within a range of $1.9 \mu_{B}-2.2 \mu_{B}$ with the rotation. Other authors also found a strong reduction of the moment in the antiferromagnetic state. For example, Kübler ${ }^{42}$ found a moment that is even slightly below $1 \mu_{B}$ in the antiferromagnetic configuration for the same lattice constant. Moruzzi and Marcus $^{43}$ obtain a reduction from $2.34 \mu_{B}$ (ferromagnetic) to $1.75 \mu_{B}$ (antiferromagnetic). Their moments are larger in both configurations, because they used a lattice constant of $a_{0}=5.48$ a.u.-i.e., $4 \%$ larger than in our calculation.

Another difference between our results and those of Stocks and co-workers is the size of $M_{\perp}(\varphi)$. These authors specify the difference between the input and output angles of the local moment rather than $M_{\perp}$. They find a maximum difference of about $25^{\circ}$. The size of the magnetic moment for that angle is about $2 \mu_{B}$, which means that $M_{\perp}$ must be about $0.9 \mu_{B}$. This value is much larger compared to the maximum $M_{\perp}$ of $0.37 \mu_{B}$ that we found. Finally, Stocks and co-workers obtain a maximum constraint field of about $0.23 \mathrm{Ry} / \mu_{B}$ at $90^{\circ}$. We also find the maximum at $90^{\circ}$, but the our value of $0.029 \mathrm{Ry} / \mu_{B}$ is almost a factor of 10 smaller. However, Fig. 6 shows that the energy difference calculated from the Hellmann-Feynman theorem using $B_{c}(\varphi)$ [panel (a), solid squares] is in fair agreement with the calculated total energy difference [panel (a), solid circles]. The Hellmann-Feynman result underestimates the energy difference between the ferromagnetic and antiferromagnetic states by about $13 \%$. Panel (a) also contains the calculated total energy of the unconstrained calculation (open circles). Naturally, the energies calculated with and without constraint are equal for the ferromagnetic and antiferromagnetic con- 


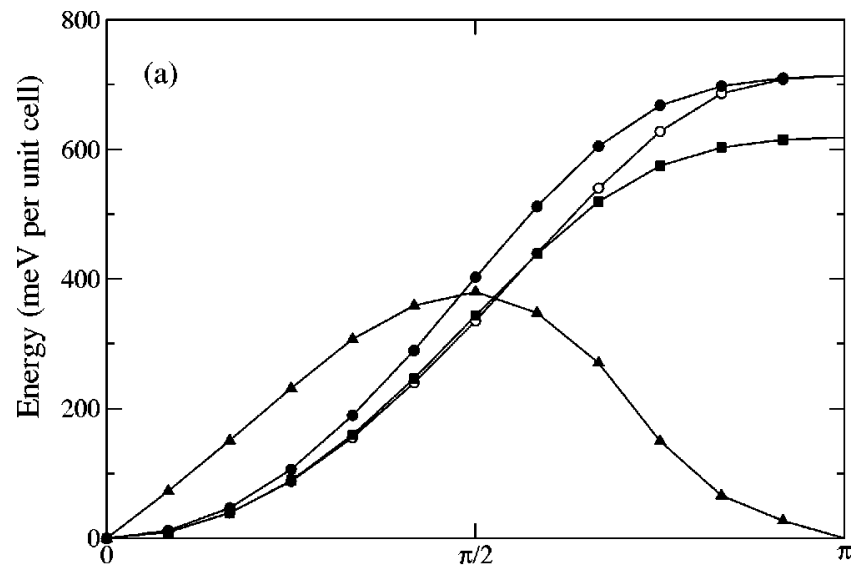

Angle of local moment $\varphi$

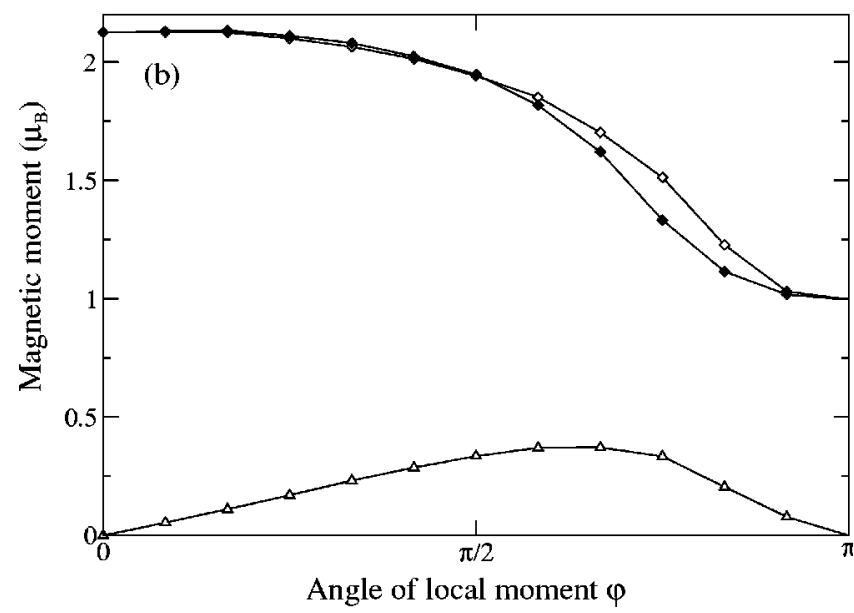

FIG. 6. (a) The total energy of bcc Fe as function of the angle of the local moment. Shown are the calculated total energies relative to the ferromagnetic energy (circles), the product of the local moment and the constraint $B$ field, $-\mu_{B} M_{\|}(\varphi) B_{c}(\varphi)$ (triangles) and integral $-\mu_{B} \int M_{\|}(\varphi) B_{c}(\varphi) d \varphi$ (squares). (b) Shown are the parallel magnetic moments $M_{\|}(\varphi)$ (diamonds) and the perpendicular moment $M_{\perp}(\varphi)$ (triangles). Both panels contain results of constrained (solid symbols) and unconstrained calculations (open symbols).

figurations, where the constraint $B$ field vanishes. For the intermediate angles the total energy calculated with constraint is always larger. We obtained the largest difference of $98 \mathrm{meV}$ at an angle of $105^{\circ}$. Compared to the other test systems this value is uncommonly large; e.g., for the $\mathrm{Cr}$ UML we found a maximum difference of only $7.4 \mathrm{meV}$. Another effect of the constraint can be seen in panel (b) of Fig. 6. In the region where the magnetic moment decreases rapidly as a function of angle, the moment of the constrained calculation (solid diamonds) is reduced even more strongly compared to the unconstrained moment (open diamonds). Other results showed that this seems to be a general trend. In situations where the magnetic moments break down rapidly, the constraint tends to reduce the moment further, while in most other instances the effect of the constraint on the size of the moment is negligible.

\section{3. fcc Fe: Spin-spiral calculations}

In order to test the spin-spiral implementation we have performed calculations on the $\gamma$-Fe (fcc Fe) system. The cal-

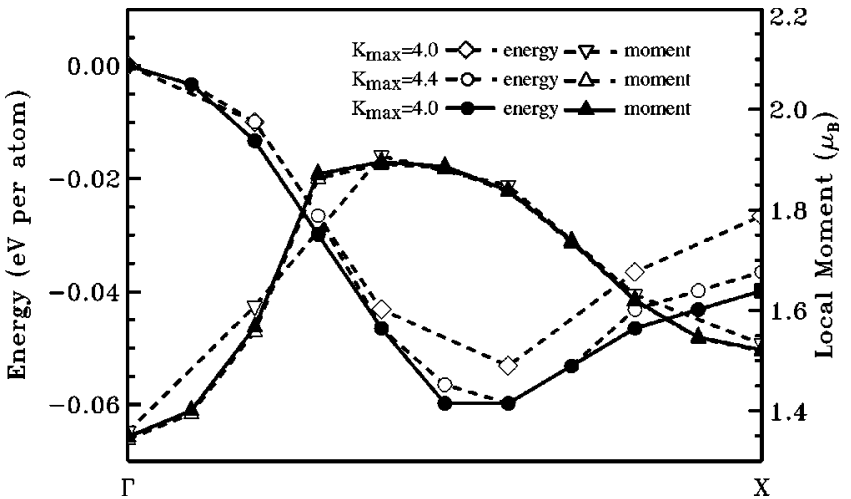

FIG. 7. Total energy and magnetic moment as a function of the spin-spiral vector q. The plot shows results of the two different implementations of the spin spirals: (i) cutoff enforced according to $|\mathbf{G}+\mathbf{k}| \leqslant K_{\max }$ (open symbols), (ii) $|\mathbf{G}+\mathbf{k} \mp q / 2| \leqslant K_{\max }$ (solid symbols). The solid square represents the energy of the layered antiferromagnetic solution calculation carried out with the collinear program.

culated total energies and magnetic moments for spin spirals with $\mathbf{q}$ vectors along the line $\overline{\Gamma X}$ are shown in Fig. 7. A spin spiral with a $\mathbf{q}$ vector at the $\Gamma$ and $X$ points corresponds to a ferromagnetic and a layered antiferromagnetic configuration, respectively. The results agree with those of previous calculations: We find the minimal energy for a $\mathbf{q}$ vector of about $\mathbf{q} \approx 0.55 \overline{\Gamma X}$. The minimizing $\mathbf{q}$ vector, the shape of the energy curve, and, in particular, the magnetic moment of $\gamma-\mathrm{Fe}$ vary strongly with the lattice parameter: For Wigner-Seitz radii of 2.66 a.u. $(a=6.81$ a.u. $)$ and smaller, Uhl et $a .^{23}$ found around the $\Gamma$ point a low-spin solution, while for Wigner-Seitz radii of 2.67 a.u. $(a=6.83$ a.u.) and larger, a high-spin solution was obtained. The magnetic moments were about $0.6 \mu_{B}$ for the low- and $2.5 \mu_{B}$ for the high-spin state. Other authors ${ }^{36}$ report a ferromagnetic moment of about $0.9 \mu_{B}$ at a Wigner-Seitz radius of $R_{W S}=2.66$ a.u. We have found a moment of $1.3 \mu_{B}$, which lies within that range. For the (experimental) $\mathrm{Cu}$ lattice constant, all authors report a total energy minimum at a $\mathbf{q}$ vector at about $0.6 \overline{\Gamma X}$, while for smaller volumes a second (local) minimum around $\mathbf{q}$ $=(0.2,0,1.0) 2 \pi / a$ develops.

We have tested two slightly different implementations of the spin spirals that use different cutoff conditions for the basis functions: (i) $|\mathbf{G}+\mathbf{k}| \leqslant K_{\max }$ and (ii) $|\mathbf{G}+\mathbf{k} \mp \mathbf{q} / 2|$ $\leqslant K_{\text {max }}$ where $\mathbf{G}$ is the wave vector of the augmented plane wave (cf. Appendix A.1). Figure 7 presents results for both implementations, implementations (i) and (ii) denoted by open and solid symbols, respectively. Implementation (i) is expected to yield less accurate energies in particular for large q. Evidence for this presumption is found in Fig. 7. Comparing for implementation (i) the results obtained for two different basis-function cutoffs $K_{\max }$ we find that the difference between the total energies increases with increasing $\mathbf{q}$ vector. The same is true when comparing implementation (i) with implementation (ii); the difference increases with the $\mathbf{q}$ vector. Even for the larger cutoff of 4.4 a.u. ${ }^{-1}$ implementation (i) deviates from implementation (ii). In particular, implementation (i) gives larger total energies. The accuracy of this 
improved implementation can be appreciated from the fact that the total energy coincides for the $\mathbf{q}$ vector at the $X$ point with the result of a collinear antiferromagnetic calculation (solid square on the right-hand side of the plot). To achieve the same accuracy with implementation (i) it would be necessary to go to even higher plane-wave cutoffs. However, increasing $K_{\max }$ from 4.0 a.u. $^{-1}$ to 4.4 a.u. $^{-1}$ means already an increase of the basis functions per atom from 85 to 115 , which makes the calculation much more time consuming, since the effort of setting up and diagonalizing the matrix scales with the number of basis functions to the third power.

\section{4. $G G A$}

We noted in Sec. II A that, in a noncollinear calculation, the gradients of the magnetization density, $m(\mathbf{r})$, that enter the GGA functional can be constructed from the vector magnetization density $\mathbf{m}(\mathbf{r})$ in two different ways: (i) we can use the gradient of the absolute value of the vector magnetization,

$$
\frac{\partial m(\mathbf{r})}{\partial x} \leftarrow \frac{\partial|\mathbf{m}(\mathbf{r})|}{\partial x},
$$

to evaluate the GGA functional. In this formulation spatial changes of the magnetization direction are not reflected; e.g., in SSDW calculations of different $\mathbf{q}$ vectors the difference in the exchange-correlation (XC) potential will enter just through changes in the absolute value of the magnetization. If $|\mathbf{m}(\mathbf{r})|$ would stay constant, the contribution of the magnetization density to the XC potential would be the same for all $\mathbf{q}$ vectors. To account approximately for changes of the magnetization direction, (ii) we can calculate the gradient of the density matrix and project it onto the direction of the magnetic moment; e.g., if $\mathbf{U}(\mathbf{r})$ diagonalizes the density matrix at $\mathbf{r}$,

$$
\frac{\partial m(\mathbf{r})}{\partial x} \leftarrow \operatorname{Tr}\left\{\boldsymbol{\sigma}_{z} \mathbf{U}(\mathbf{r})^{\dagger} \frac{\partial \boldsymbol{\rho}(\mathbf{r})}{\partial x} \mathbf{U}(\mathbf{r})\right\}
$$

The latter implementation was also chosen by Knöpfle et $a l^{40}{ }^{40}$ who investigated the spin-spiral ground state of $\gamma$-Fe by a modified augmented spherical wave (MASW) method applying the Perdew-Burke-Ernzerhof (PBE) form ${ }^{44}$ of the GGA. Körling and Ergon ${ }^{39}$ used the older PW91 form ${ }^{45}$ within the atomic sphere approximation. To sort out the differences between these two exchange-correlation potentials, we calculated the energy of fcc Fe with a lattice constant of 6.70 a.u. as a function of the $\mathbf{q}$ vector for both forms of the GGA. The results are summarized in Fig. 8(a), from which we see that the major difference between the two forms of the GGA is the enhancement of the magnetic moments in the PBE form. In both forms we find two minima of the energy at $\mathbf{q} \approx 0.6 \overline{\Gamma X}$ and $\mathbf{q} \approx 0.2 \overline{X W}$. The PBE form tends to stabilize the former minimum with respect to the latter by 1.5 meV/atom and the energy differences are nowhere larger than $5 \mathrm{meV} / \mathrm{atom}$.

The influence of the choice of implementation of the gradients [i.e., according to Eq. (27) or Eq. (28)], presented in Fig. $8(\mathrm{~b})$, is even smaller. The largest difference can be de-

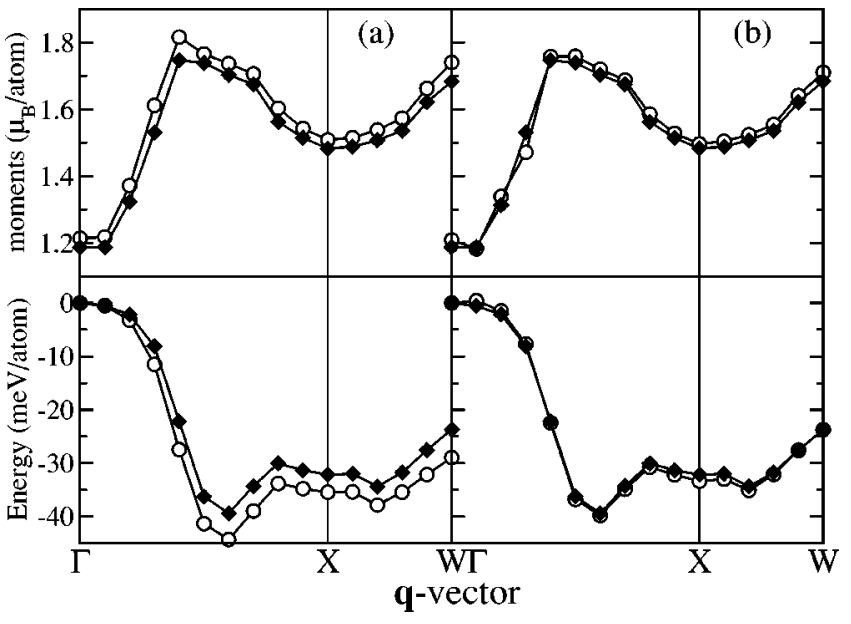

FIG. 8. The magnetic moments (top) and energy (bottom) of the spin spiral of $\gamma$-Fe as a function of the $\mathbf{q}$ vector (a) calculated with the PBE (Ref. 44) (open circles) and the PW91 (Ref. 45) (solid diamonds) form of the GGA and (b) with gradients according to Eq. (27) (solid diamonds) or Eq. (28) (open circles). Panel (a) was calculated according to Eq. (27), panel (b) with the PW91 form of the GGA. The energies are given relative to the energy of the ferromagnetic state.

tected at the $X$ point, but even here the energy difference is smaller than $1.2 \mathrm{meV} / \mathrm{atom}$. One should mention here that for the calculation of the gradient of the magnetization density $\boldsymbol{\nabla} m(\mathbf{r})$, the most significant difference between these two implementations can be expected from regions where the magnetization density changes its sign: then, in the case of a linear behavior of the component of $\mathbf{m}(\mathbf{r})$ that changes sign, Eq. (27) introduces an artificial minimum in the absolute value of the gradient of the magnetization density, $|\nabla m(\mathbf{r})|$, while Eq. (28) avoids this artifact. Therefore, even in a collinear structure, the results obtained by the two equations can differ, as can be seen at the $X$ point in Fig. 8 .

\section{Intra-atomic noncollinearity}

In their papers both Knöpfle et al. ${ }^{40}$ and Hobbs and Hafner ${ }^{31}$ stress the importance of intra-atomic noncollinearity. With the choice of different muffin-tin radii, we can vary the fraction of space in which the magnetic field is treated as a vector quantity. From Fig. 2 we see that (at least for $\mathrm{Cr}$ ) the closer we come to the nucleus, the smaller the noncollinear effects get. Therefore, we expect that choosing touching muffin-tin radii (in our fcc Fe example this would correspond to 2.36 a.u.) might create the largest deviations, but with shrinking radii the results should converge rather quickly. The results of these calculations are shown in Fig 9. With shrinking muffin-tin radius, the magnetic moments get generally smaller (since they are evaluated as the integral of the magnetization density inside the muffin-tin spheres); otherwise there are no dramatic effects to be observed. Looking at the energies, we see that for muffin-tin radii of 2.3 and 2.1 a.u. the energies differ at most by $1.4 \mathrm{meV} /$ atom (at the $X$ point) while in a comparison of the calculations with muffintin radii of 2.1 and 1.9 a.u. this value decreased to a mere 1.0 meV/atom. Keeping in mind that at the $X$ point all total en- 


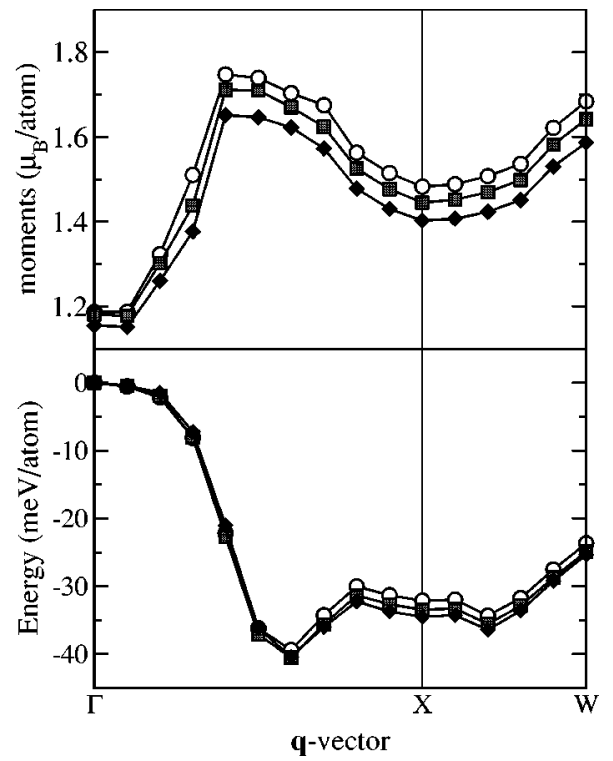

FIG. 9. The magnetic moments (top) and energy (bottom) of the spin spiral of $\gamma$-Fe as a function of the $\mathbf{q}$ vector calculated with muffin-tin radii of 2.3 a.u. (open circles), 2.1 a.u. (shaded squares), and 1.9 a.u. (solid diamonds). The energies are given relative to the energy of the ferromagnetic state. Note that the magnetic moments are quantities integrated within the muffin-tin spheres. The planewave cutoff for these calculations was chosen to keep the product of muffin-tin radius and $K_{\max }$ approximately constant (9.6). The PW91 (Ref. 45) form of the GGA with gradients according to Eq. (27) was used.

ergy differences are caused by the change of the muffin-tin radius and the plane-wave cutoff (cf. also Sec. III B 3), we suspect that most of the (although small) energy differences observed in Fig. 9 are not associated with intra-atomic noncollinearity.

Sjöstedt and Nordström ${ }^{11}$ also investigated the influence of the intra-atomic noncollinearity in this system and found the largest contributions in a region around $\mathbf{q} \approx 0.3 \overline{\Gamma X}$. Allowing for intra-atomic noncollinearity they find an almost linear decrease between $\Gamma$ and $0.5 \overline{\Gamma X}$, while Knöpfle et $a l .{ }^{40}$ and (although not for exactly the same volume) Marsman and Hafner ${ }^{41}$ find a shape similar to Fig. 9. Comparing these different calculations, we find it difficult to sort out the influences of the different implementations. Staying within our approximation we observe that a reduction of the area covered by the muffin tins by $44 \%$ leads to no significant change of the result. Nevertheless, we want to mention that for heavy elements (e.g., the actinides ${ }^{6}$ ) and for all problems where spin-orbit coupling is important ${ }^{12}$ intra-atomic noncollinearity cannot be neglected.

\section{ACKNOWLEDGMENTS}

The authors gratefully acknowledge support from TMR network Contract No. FMRX-CT-0178. Ph.K. and S.B. thank L. Sandratskii for fruitful discussions and acknowledge the hospitality of the University of Uppsala. L.N. is grateful to the support from the Swedish Natural Science Research Council (NFR).

\section{APPENDIX: IMPLEMENTATION OF NONCOLLINEAR MAGNETISM IN THE FLAPW METHOD}

\section{Noncollinear magnetism in the FLAPW method}

The FLAPW method for collinear calculations uses two sets of radial basis functions inside the muffin tins for the spin directions. For each spin direction they are set up using the spherical part of the corresponding potential $V_{\uparrow}(r)$ or $V_{\downarrow}(r)$ and energy parameter $\varepsilon_{l \uparrow}$ or $\varepsilon_{l \downarrow}$. In the noncollinear case it is still possible to work with $V_{\uparrow}(r)$ and $V_{\downarrow}(r)$, since we restrict the magnetization to the local quantization axis. Therefore, a local spin-space coordinate frame is introduced with the $z$ axis parallel to the local quantization axis. $V_{\uparrow}$ and $V_{\downarrow}$ are now spin up and down with respect to the local axis. Since both the potential and basis functions are set up in terms of the local spin coordinate frame, the determination of the basis functions and calculation of the integrals of these functions with the Hamiltonian inside the muffin-tin spheres are completely unchanged. The changes come in when the basis functions inside the muffin tins are matched to the plane waves in the interstitial region, because the local spin coordinate frame is rotated with respect to the global frame.

The FLAPW method uses augmented plane waves as basis functions. Therefore, each basis function can be uniquely identified by its wave vector $\mathbf{G}$ and the spin direction. The basis functions in the interstitial region are

$$
\boldsymbol{\varphi}_{\mathbf{G}, \sigma}(\mathbf{k}, \mathbf{r})=e^{i(\mathbf{k}+\mathbf{G}) \cdot \mathbf{r}} \chi_{\sigma}^{g} .
$$

$\chi_{\sigma}^{g}$ is a two-component spinor. The index $g$ has been added to signify that $\chi_{\sigma}^{g}$ is the representation of this spinor in the global spin frame. In this global frame the $\chi_{\sigma}^{g}$ are just the regular two-component spinors $\chi_{\uparrow}^{g}=\left(\begin{array}{l}1 \\ 0\end{array}\right)$ and $\chi_{\downarrow}^{g}=\left(\begin{array}{l}0 \\ 1\end{array}\right)$ used also in collinear calculations. In difference to collinear calculations, in noncollinear calculations, the potential matrix $\mathbf{V}$ - and thus the Hamiltonian-is not anymore diagonal in the two-dimensional spin space. In the vacuum region, we also use the global spin frame for the representation of the basis functions. Only inside the muffin tin spheres is the basis set changed, because we use a local spin coordinate frame, which is rotated with respect to the global frame. Thus, the basis set has the following form:

$$
\boldsymbol{\varphi}_{\mathbf{G}, \sigma}(\mathbf{k}, \mathbf{r})= \begin{cases}e^{i(\mathbf{G}+\mathbf{k}) \mathbf{r}} \chi_{\sigma}^{g}, & \text { int., } \\ {\left[A_{\sigma}^{\mathbf{G}}\left(\mathbf{k}_{\|}\right) u_{\sigma}^{\mathbf{G}_{\|}}\left(\mathbf{k}_{\|}, z\right)+B_{\sigma}^{\mathbf{G}}\left(\mathbf{k}_{\|}\right) \dot{u}_{\sigma}^{\mathbf{G}_{\|}}\left(\mathbf{k}_{\|}, z\right)\right] e^{i\left(\mathbf{G}_{\|}+\mathbf{k}_{\|}\right) \mathbf{r}_{\|}} \chi_{\sigma}^{g},} & \text { vac. }, \\ \sum_{\sigma^{\alpha}} \sum_{L}\left[A_{L \sigma \sigma^{\alpha}}^{\alpha \mathbf{G}}(\mathbf{k}) u_{\ell \sigma^{\alpha}}^{\alpha}(r)+B_{L \sigma \sigma^{\alpha}}^{\alpha \mathbf{G}}(\mathbf{k}) \dot{u}_{\ell \sigma^{\alpha}}^{\alpha}(r)\right] Y_{L}(\hat{\mathbf{r}}) \chi_{\sigma^{\alpha}}, & \operatorname{MT} \alpha,\end{cases}
$$


where $\mathbf{k}$ is the Bloch vector, $\mathbf{G}$ is a reciprocal lattice vector, $L$ abbreviates the quantum numbers $l$ and $m$, and $\|$ denotes the components parallel to the surface, if any. At the boundary of the muffin-tin spheres $\alpha$, the plane waves are matched to solutions of the (scalar-relativistic) radial Schrödinger equation $u_{l \sigma}^{\alpha}\left(r, \varepsilon_{l \sigma}\right)$ and its energy derivative $\dot{u}_{l \sigma}^{\alpha}\left(r, \varepsilon_{l \sigma}\right)$ $=\partial u_{l \sigma}^{\alpha}\left(r, \varepsilon_{l \sigma}\right) / \partial \varepsilon$ via the matching coefficients $A$ and $B$ :

$$
\begin{aligned}
\boldsymbol{\varphi}_{\mathbf{G}, \sigma}(\mathbf{k}, \mathbf{r})= & \sum_{\sigma^{\alpha}} \varphi_{\mathbf{G}, \sigma^{\alpha}}^{\sigma}(\mathbf{k}, \mathbf{r}) \chi_{\sigma^{\alpha}}=\sum_{\sigma^{\alpha}} \sum_{L}\left[A_{L \sigma \sigma^{\alpha}}^{\alpha \mathbf{G}, \mathbf{k}} u_{l \sigma^{\alpha}}^{\alpha}(r)\right. \\
& \left.+B_{L \sigma \sigma^{\alpha}}^{\alpha \mathbf{G}, \mathbf{k}} u_{l \sigma^{\alpha}}^{\alpha}(r)\right] Y_{L}(\hat{\mathbf{r}}) \chi_{\sigma^{\alpha}},
\end{aligned}
$$

where the sum is over the local spin directions $\sigma^{\alpha}$. As a consequence, when the functions in the sphere are matched to the plane waves at the boundary of the muffin-tin spheres, each spin direction in the interstitial region is matched to both the spin-up and -down basis functions in the sphere. The noncollinear $A$ and $B$ coefficients can be expressed in terms of the collinear coefficients:

$$
A_{L \sigma \sigma^{\alpha}}^{\alpha \mathbf{G}, \mathbf{k}}=\left[\left(\chi_{\sigma^{\alpha}}^{\alpha g}\right)^{*} \chi_{\sigma}\right] A_{L \sigma}^{\alpha \mathbf{G}, \mathbf{k}}
$$

(the same holds for the $B$ coefficients) with spinors

$$
\chi_{\uparrow}^{\alpha g}=\left(\begin{array}{c}
e^{-i \frac{\varphi}{2} \cos \left(\frac{\vartheta}{2}\right)} \\
e^{i \frac{\varphi}{2} \sin \left(\frac{\vartheta}{2}\right)}
\end{array}\right), \quad \chi_{\downarrow}^{\alpha g}=\left(\begin{array}{c}
-e^{-i \frac{\varphi}{2} \sin \left(\frac{\vartheta}{2}\right)} \\
e^{i \frac{\varphi}{2} \cos \left(\frac{\vartheta}{2}\right)}
\end{array}\right)
$$

Here, the angles $\varphi$ and $\vartheta$ define the direction of the magnetic moment of atom $\alpha$ with respect to the global frame. With these relations, the Hamiltonian matrix elements of a noncollinear calculation can be related to that of a collinear calculation, $H_{M T}^{\mathbf{G}^{\prime} \mathbf{G}}(\mathbf{k})$, by

$$
H_{M T^{\alpha}}^{\mathbf{G}^{\prime} \sigma^{\prime} \mathbf{G} \sigma}(\mathbf{k})=\sum_{\sigma^{\alpha}}\left[\left(\chi_{\sigma^{\alpha}}^{\alpha g}\right)^{\alpha g} \chi_{\sigma^{\prime}}\right]^{*}\left[\left(\chi_{\sigma^{\alpha}}^{\alpha g}\right)^{*} \chi_{\sigma}\right] H_{M T^{\alpha} \sigma^{\alpha}}^{\mathbf{G}^{\prime} \mathbf{G}}(\mathbf{k})
$$

and a similar relation holds for the overlap matrix. Since all quantities defined inside the muffin-tin spheres can be related to those of a collinear calculation by applying a spin-rotation matrix, the combination of this method with local orbitals ${ }^{46}$ and the $\mathrm{LDA}+\mathrm{U}$ formalism ${ }^{47}$ is rather straightforward.

In the interstitial and vacuum region we work within the global spin coordinate frame. Using the step function $\Theta$, which "cuts out" the muffin-tin spheres, the Hamiltonian matrix can be set up directly to give

$H_{I N T}^{\mathbf{G} \sigma \mathbf{G}^{\prime} \boldsymbol{\sigma}^{\prime}}(\mathbf{k})=\left(V_{\sigma \sigma^{\prime}} \Theta\right)_{\left(\mathbf{G}-\mathbf{G}^{\prime}\right)}+\delta_{\sigma \sigma^{\prime}} \frac{\hbar^{2}}{2 m}\left(\mathbf{G}^{\prime}+\mathbf{k}\right)^{2} \Theta_{\left(\mathbf{G}-\mathbf{G}^{\prime}\right)}$,

while the overlap matrix is diagonal in spin space with the diagonal elements the same as in the collinear case.

\section{Constraints in the FLAPW method}

In the local spin coordinate frame the additional contribution to the Hamiltonian matrix due to the constraint $B$ field in the sphere is purely off diagonal. If we calculate a matrix element of the Hamiltonian [Eq. (19)] with the basis functions [Eq. (A2)], we get

$$
H_{M T^{\alpha}}^{\mathbf{G}^{\prime} \sigma^{\prime} \mathbf{G} \sigma}(\mathbf{k})=\sum_{\sigma^{\alpha}}\left\langle\varphi_{\mathbf{G}^{\prime}, \sigma^{\alpha}}^{\sigma^{\prime}}(\mathbf{k})\left|\mathcal{H}_{M T^{\alpha}}^{\text {off-d }}\right| \varphi_{\mathbf{G}, \sigma^{\alpha}}^{\sigma}(\mathbf{k})\right\rangle .
$$

Substituting Eq. (A4) yields

$$
\begin{aligned}
H_{M T^{\alpha}}^{\mathbf{G}^{\prime} \sigma^{\prime} \mathbf{G} \sigma}(\mathbf{k})= & -\mu_{B} \sum_{\sigma^{\alpha}}\left(B_{c, x}^{\alpha}+\operatorname{sgn}\left(\sigma^{\alpha}\right) i B_{c, y}^{\alpha}\right) \\
& \times\left[\left(\chi_{-\sigma^{\alpha}}^{\alpha g}\right)^{*} \chi_{\sigma^{\prime}}\right]^{*}\left[\left(\chi_{\sigma^{\alpha}}^{\alpha g}\right)^{*} \chi_{\sigma}\right] \\
& \times\left\langle\varphi_{\mathbf{G}^{\prime}, \sigma^{\alpha}}^{\sigma^{\prime}}(\mathbf{k}) \mid \varphi_{\mathbf{G}, \sigma^{\alpha}}^{\sigma}(\mathbf{k})\right\rangle .
\end{aligned}
$$

\section{Spin spirals in the FLAPW method}

As suggested by Eq. (18), the plane wave basis set [Eq. (A1)] used in the interstitial region can be written as

$$
\boldsymbol{\varphi}_{\mathbf{G}, \sigma}(\mathbf{k}, \mathbf{q}, \mathbf{r})=e^{i(\mathbf{G}+\mathbf{k} \mp \mathbf{q} / 2) \cdot \mathbf{r}} \chi_{\sigma},
$$

where the minus sign in $\mp \mathbf{q} / 2$ holds for spin up and the plus sign hold for spin down. Inside the muffin tins the changes to the basis set enter only through the boundary conditions, since the functions inside the spheres need to be matched to the plane waves in the interstitial region including the extra term $\mp \mathbf{q} / 2$ now. Replacing $e^{i(\mathbf{G}+\mathbf{k}) \cdot \mathbf{r}}$ by $e^{i(\mathbf{G}+\mathbf{k}+\mathbf{q} / 2) \cdot \mathbf{r}}$ in the matching condition, we find that the $A$ and $B$ coefficients of a spin-spiral calculation can be expressed in terms of the corresponding coefficients of a collinear calculation,

$$
A_{L \sigma \sigma^{\alpha}}^{\alpha \mathbf{G}}(\mathbf{k}, \mathbf{q})=\left[\left(\chi_{\sigma^{\alpha}}^{\alpha g}\right)^{*} \chi_{\sigma}\right] A_{L \sigma}^{\alpha \mathbf{G}}(\mathbf{k} \mp \mathbf{q} / 2),
$$

and the same holds, of course, for the $B$ coefficients. When local orbitals are used in a calculation, care has to be taken to ensure that the local orbital is "attached" to the same $\mathbf{G}$ vector in the majority $(\mathbf{G}+\mathbf{k}-\mathbf{q} / 2)$ and minority $(\mathbf{G}+\mathbf{k}$ $+\mathbf{q} / 2)$ spin channels. Also here combination with the $\mathrm{LDA}+\mathrm{U}$ formalism is straightforward and has been used in combination with local orbitals successfully for the determination of the magnetic ground state of Eu. ${ }^{48}$ In contrast to an ordinary noncollinear calculation the coefficients [Eq. (A11)] for the two interstitial spin directions differ by more than a complex prefactor in the spin-spiral case. Therefore, the very simple relations for the Hamiltonian and overlap matrix elements [Eq. (A6)] do not hold anymore. Instead, the Hamiltonian matrix elements have to calculated directly from

$$
\begin{aligned}
& H_{M T^{\alpha}}^{\mathbf{G}^{\prime} \sigma^{\prime} \mathbf{G} \sigma}(\mathbf{k}, \mathbf{q}) \\
& \quad=\sum_{\sigma^{\alpha}}\left\langle\varphi_{\mathbf{G}^{\prime}, \sigma^{\alpha}}^{\sigma^{\prime}}(\mathbf{k}, \mathbf{q}) \chi_{\sigma^{\alpha}}^{\alpha g}\left|\mathcal{H}_{M T^{\alpha}}\right| \varphi_{\mathbf{G}, \sigma^{\alpha}}^{\sigma}(\mathbf{k}, \mathbf{q}) \chi_{\sigma^{\alpha}}^{\alpha g}\right\rangle .
\end{aligned}
$$


As a consequence, the numerical effort setting up the contribution to the Hamiltonian and overlap matrix from the muffin tins is increased compared to an ordinary noncollinear calculation.

When the cone angle $\vartheta^{\alpha}$ [cf. Eq. (14)] of the spin spiral is not a multiple of $\pi / 2$, the spiral-spin-density wave is not necessarily a stationary solution and either the ground-state cone angle can be determined, or a constraint has to be used. The constraining field is then, of course, in itself modulated by the same $\mathbf{q}$ vector as the spin spiral.
*Electronic address: G.Bihlmayer@fz-juelich.de

${ }^{1}$ For an overview see L.M. Sandratskii, Adv. Phys. 47, 91 (1998).

${ }^{2}$ U. von Barth and L. Hedin, J. Phys. C 5, 1629 (1972).

${ }^{3}$ L. Sandratskii and P. Guletskii, J. Phys. F: Met. Phys. 16, L43 (1986).

${ }^{4}$ J. Kübler, K. Höck, J. Sticht, and A. Williams, J. Phys. F: Met. Phys. 18, 469 (1988).

${ }^{5}$ O. Mryasov, A. Lichtenstein, L. Sandratskii, and V. Gubanov, J. Phys. C 3, 7683 (1991).

${ }^{6}$ L. Nordström and D.J. Singh, Phys. Rev. Lett. 76, 4420 (1996).

${ }^{7}$ T. Oda, A. Pasquarello, and R. Car, Phys. Rev. Lett. 80, 3622 (1998).

${ }^{8}$ D.M. Bylander and L. Kleinman, Phys. Rev. B 58, 9207 (1998).

${ }^{9}$ O. Ivanov and V. Antropov, J. Appl. Phys. 85, 4821 (1999).

${ }^{10}$ D. Hobbs, G. Kresse, and J. Hafner, Phys. Rev. B 62, 11556 (2000).

${ }^{11}$ E. Sjöstedt and L. Nordström, Phys. Rev. B 66, 014447 (2002).

${ }^{12}$ K. Nakamura, T. Ito, A.J. Freeman, L. Zhong, and J. Fernandezde-Castro, Phys. Rev. B 67, 014420 (2003).

${ }^{13} \mathrm{Ph}$. Kurz, G. Bihlmayer, K. Hirai, and S. Blügel, Phys. Rev. Lett. 86, 1106 (2001)

${ }^{14} \mathrm{Ph}$. Kurz, G. Bihlmayer, K. Hirai, and S. Blügel, Phase Transitions 75, 101 (2002).

${ }^{15}$ V.P. Antropov, M.I. Katsnelson, M. van Schilfgaarde, and B.N. Harmon, Phys. Rev. Lett. 75, 729 (1995).

${ }^{16}$ V.P. Antropov, M.I. Katsnelson, B.N. Harmon, M. van Schilfgaarde, and D. Kusnezov, Phys. Rev. B 54, 1019 (1996).

${ }^{17}$ Y. Tsunoda, J. Phys.: Condens. Matter 1, 10427 (1989).

${ }^{18}$ G. Venturini, R. Welter, E. Ressouche, and B. Malaman, J. Alloys Compd. 210, 213 (1994).

${ }^{19}$ P.H. Dederichs, S. Blügel, R. Zeller, and H. Akai, Phys. Rev. Lett. 53, 2512 (1984).

${ }^{20}$ G. Stocks, B. Ujfalussy, X.-D. Wang, D. Nicholson, W. Shelton, Y. Wang, A. Canning, and B. Györffy, Philos. Mag. B 78, 665 (1998).

${ }^{21}$ B. Ujfalussy, X.-D. Wang, D. Nicholson, W. Shelton, G. Stocks, Y. Wang, and B. Györffy, J. Appl. Phys. 85, 4824 (1999).

${ }^{22}$ L. Nordström and A. Mavromaras, Europhys. Lett. 49, 775 (2000).

${ }^{23}$ M. Uhl, L. Sandratskii, and J. Kübler, J. Magn. Magn. Mater. 103, 314 (1992).

${ }^{24}$ C. Herring, in Magnetism, edited by G. Rado and H. Suhl (Academic, New York, 1966).

${ }^{25}$ L. Sandratskii, Phys. Status Solidi B 136, 167 (1986).

${ }^{26}$ N. Ashcroft and N. Mermin, Solid State Physics (Saunders College, Philadelphia, 1976).
${ }^{27}$ E. Wimmer, H. Krakauer, M. Weinert, and A.J. Freeman, Phys. Rev. B 24, 864 (1981).

${ }^{28}$ M. Weinert, E. Wimmer, and A.J. Freeman, Phys. Rev. B 26, 4571 (1982).

${ }^{29}$ V. Moruzzi, J. Janak, and A. Williams, Calculated Electronic Properties of Metals (Pergamon, New York, 1978).

${ }^{30}$ S. Heinze, P. Kurz, D. Wortmann, G. Bihlmayer, and S. Blügel, Appl. Phys. A: Mater. Sci. Process. 75, 25 (2002).

${ }^{31}$ D. Hobbs and J. Hafner, J. Phys.: Condens. Matter 12, 7025 (2000).

${ }^{32}$ A. Oswald, R. Zeller, and P. Dederichs J. Magn. Magn. Mater. 54-57, 1247 (1986).

${ }^{33}$ R. Yu, D. Singh, and H. Krakauer, Phys. Rev. B 43, 6411 (1991).

${ }^{34}$ O. Grotheer and M. Fähnle, Phys. Rev. B 59, 13965 (1998).

${ }^{35}$ D. Crockford, D. Bird, and M. Long, J. Phys.: Condens. Matter 3, 8665 (1991).

${ }^{36}$ O.N. Mryasov, V.A. Gubanov, and A.I. Liechtenstein, Phys. Rev. B 45, 12330 (1992).

${ }^{37}$ M. Uhl, L.M. Sandratskii, and J. Kübler, Phys. Rev. B 50, 291 (1994).

${ }^{38}$ O. Mryasov, A. Freeman, and A. Lichtenstein, J. Appl. Phys. 79, 4805 (1996).

${ }^{39}$ M. Körling and J. Ergon, Phys. Rev. B 54, 8293 (1996).

${ }^{40}$ K. Knöpfle, L.M. Sandratskii, and J. Kübler, Phys. Rev. B 62, 5564 (2000).

${ }^{41}$ M. Marsman and J. Hafner, Phys. Rev. B 66, 224409 (2002).

${ }^{42}$ J. Kübler, Phys. Lett. 81A, 81 (1981).

${ }^{43}$ V.L. Moruzzi and P.M. Marcus, Phys. Rev. B 42, 8361 (1990).

${ }^{44}$ J.P. Perdew, K. Burke, and M. Ernzerhof, Phys. Rev. Lett. 77, 3865 (1996).

${ }^{45}$ J.P. Perdew, J.A. Chevary, S.H. Vosko, K.A. Jackson, M.R. Pederson, D.J. Singh, and C. Fiolhais, Phys. Rev. B 46, 6671 (1992).

${ }^{46}$ D. Singh, Phys. Rev. B 43, 6388 (1991).

${ }^{47}$ V.I. Anisimov, F. Aryasetiawan, and A.I. Lichtenstein, J. Phys.: Condens. Matter 9, 767 (1996).

${ }^{48}$ G. Bihlmayer, Ph. Kurz, and S. Blügel (unpublished).

${ }^{49}$ The factor $\mu_{B}$, which was already present in Eq. (7), appears in the Hellmann-Feynman theorem because of the definition of the magnetization density and the magnetic moment. In the context of ab initio calculations the ( $z$ component of the) magnetization density is commonly defined as $m_{z}=n_{\uparrow}-n_{\downarrow}$, and we define the density matrix [Eq. (1)] in that spirit. However, the standard definition of the magnetization density in electrodynamic is $m_{z}$ $=-\mu_{B}\left(n_{\uparrow}-n_{\downarrow}\right)$. 